\title{
On the KP I Transonic Limit of Two-Dimensional Gross-Pitaevskii Travelling Waves
}

Fabrice Béthuel, Philippe Gravejat, and Jean-Claude Saut

Communicated by $Y$. Charles Li, received June 6, 2008.

\begin{abstract}
We provide a rigorous mathematical derivation of the convergence in the long-wave transonic limit of the minimizing travelling waves for the two-dimensional Gross-Pitaevskii equation towards ground states for the Kadomtsev-Petviashvili equation (KP I).
\end{abstract}

\section{Contents}

1. Introduction 241

2. Some properties of solitary wave solutions to (KP I) 248

3. Some properties of solutions to (TWc) 252

4. (TWc) in the slow space variables 256

5. Properties of the kernels $K_{\varepsilon}^{i, j} \quad 264$

6. Sobolev bounds for $N_{\mathfrak{p}}$ and $\Theta_{\mathfrak{p}} \quad 266$

7. Convergence towards (KP I) 273

$\begin{array}{ll}\text { References } & 279\end{array}$

\section{Introduction}

1.1. Statement of the results. The Gross-Pitaevskii equation

$$
i \partial_{t} \Psi=\Delta \Psi+\Psi\left(1-|\Psi|^{2}\right) \text { on } \mathbb{R}^{N} \times \mathbb{R},
$$

appears as a relevant model in various areas of physics: Bose-Einstein condensation, fluid mechanics (see e.g. $[\mathbf{1 3}, \mathbf{2 7}, \mathbf{1 9}, \mathbf{8}]$ ), nonlinear optics (see e.g. $[\mathbf{2 3}]$ )... At least on a formal level, this equation is hamiltonian, with a conserved Hamiltonian

1991 Mathematics Subject Classification. 35, 76.

Key words and phrases. Transonic limit, Kadomtsev-Petviashvili equation, Gross-Pitaevskii equation, travelling waves.

(c)2008 International Press 
given by the Ginzburg-Landau energy,

$$
E(\Psi)=\frac{1}{2} \int_{\mathbb{R}^{N}}|\nabla \Psi|^{2}+\frac{1}{4} \int_{\mathbb{R}^{N}}\left(1-|\Psi|^{2}\right)^{2} \equiv \int_{\mathbb{R}^{N}} e(\Psi) .
$$

Note that the boundedness of the Ginzburg-Landau energy implies that in some sense,

$$
|\Psi(x, \cdot)| \rightarrow 1, \text { as }|x| \rightarrow+\infty .
$$

As a matter of fact, this condition provides a richer dynamics than in the case of null condition at infinity which is essentially governed by dispersion and scattering. In particular, (GP) has nontrivial coherent localized structures called travelling waves.

The existence of finite energy travelling waves was addressed and established in

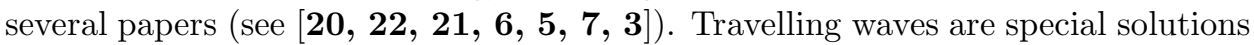
to (GP) of the form

$$
\Psi(x, t)=u\left(x_{1}-c t, x_{\perp}\right), x_{\perp}=\left(x_{2}, \ldots, x_{N}\right) .
$$

They are supposed to play an important role in the full dynamics of (GP). The equation for the profile $u$ is given by

$$
i c \partial_{1} u+\Delta u+u\left(1-|u|^{2}\right)=0 .
$$

The parameter $c \in \mathbb{R}$ corresponds to the speed of the travelling waves. We may restrict to the case $c \geq 0$. Indeed, when $u$ is a travelling wave of speed $c$, the map $\bar{u}$ obtained by complex conjugation is a travelling wave of speed $-c$.

The existence of solutions to (TWc) was obtained in the above quoted papers through variational arguments, namely minimization under constraints $[\mathbf{5}, \mathbf{3}]$, or mountain-pass theorems $[\mathbf{6}, \mathbf{7}]$. In dimensions two and three, a full branch of solutions is constructed in $[\mathbf{3}]$ minimizing the Ginzburg-Landau energy $E$ under fixed momentum $p$. In this context, the momentum is defined by

$$
p(u)=\frac{1}{2} \int_{\mathbb{R}^{N}}\left\langle i \partial_{1} u, u-1\right\rangle .
$$

This integral quantity is also formally conserved by (GP). A notable difficulty in the variational approach is to give a meaning to the momentum in the space of maps of finite Ginzburg-Landau energy (see e.g. [2, 4]). However, the momentum is well-defined for finite energy travelling wave solutions. Indeed, it is proved in [16] that they belong to the space $W\left(\mathbb{R}^{N}\right)$, defined as

$$
W\left(\mathbb{R}^{N}\right)=\{1\}+V\left(\mathbb{R}^{N}\right)
$$

where we have set

$$
\begin{aligned}
V\left(\mathbb{R}^{N}\right)= & \left\{v: \mathbb{R}^{N} \mapsto \mathbb{C}, \text { s.t. }(\nabla v, \operatorname{Re}(v)) \in L^{2}\left(\mathbb{R}^{N}\right)^{2},\right. \\
& \left.\operatorname{Im}(v) \in L^{4}\left(\mathbb{R}^{N}\right), \text { and } \nabla \operatorname{Re}(v) \in L^{\frac{4}{3}}\left(\mathbb{R}^{N}\right)\right\} .
\end{aligned}
$$

Separating real and imaginary parts, a direct computation shows that the quantity $\left\langle i \partial_{1} v, v-1\right\rangle$ is integrable for any function $v \in W\left(\mathbb{R}^{N}\right)$, so that the momentum of travelling wave solutions is well-defined.

The main focus of this paper is a qualitative description of small GinzburgLandau energy solutions in the two-dimensional case. Such solutions are known to exist in view of the following result. 
THEOREM $1([\mathbf{3}])$. i) Let $\mathfrak{p}>0$. There exists a non-constant finite energy solution $u_{\mathfrak{p}} \in W\left(\mathbb{R}^{2}\right)$ to $(\mathrm{TWc})$, with $0<c=c\left(u_{\mathfrak{p}}\right)<\sqrt{2}$, and

$$
p\left(u_{\mathfrak{p}}\right) \equiv \frac{1}{2} \int_{\mathbb{R}^{2}}\left\langle i \partial_{1} u_{\mathfrak{p}}, u_{\mathfrak{p}}-1\right\rangle=\mathfrak{p}
$$

such that $u_{\mathfrak{p}}$ is solution to the minimization problem

$$
E\left(u_{\mathfrak{p}}\right)=E_{\min }(\mathfrak{p})=\inf \left\{E(v), v \in W\left(\mathbb{R}^{2}\right), p(v)=\mathfrak{p}\right\} .
$$

ii) There exist some positive constants $K_{0}, K_{1}$ and $\mathcal{S}_{K P}$, not depending on $\mathfrak{p}$, such that we have the asymptotic behaviours

$$
0<\frac{48 \sqrt{2}}{\mathcal{S}_{K P}^{2}} \mathfrak{p}^{3}-K_{0} \mathfrak{p}^{4} \leq \sqrt{2} \mathfrak{p}-E\left(u_{\mathfrak{p}}\right) \leq K_{1} \mathfrak{p}^{3}
$$

for any $\mathfrak{p}$ sufficiently small.

A more precise definition of the constant $\mathcal{S}_{K P}$ will be provided in the course of our discussion of the Kadomtsev-Petviashvili equation (KP I). It should be noticed that we have, in view of $(3)$,

$$
E\left(u_{\mathfrak{p}}\right) \sim \sqrt{2} \mathfrak{p},
$$

for small values of the momentum $\mathfrak{p}$, so that Theorem 1 provides a branch of travelling wave solutions with arbitrary small energy. Our aim is to describe the asymptotic behaviour, as $\mathfrak{p} \rightarrow 0$, of the solutions $u_{\mathfrak{p}}$ constructed above.

We recall that, in view of $[\mathbf{6}, \mathbf{1 5}, \mathbf{1 7}]$, any finite energy travelling waves are subsonic in dimension two, i.e. any non-constant finite energy solution $v$ to (TWc) satisfies

$$
0<|c(v)|<\sqrt{2} .
$$

The speed $\sqrt{2}$ corresponds to the speed of sound waves at infinity around the constant solution $\Psi=1$ to (GP). Moreover, the quantity

$$
\varepsilon(v)=\sqrt{2-c(v)^{2}}
$$

is related to the energy $E(v)$ and the uniform norm of $1-|v|$ as follows.

Proposition $1([\mathbf{3}])$. Let $v$ be a non-constant finite energy solution to (TWc) on $\mathbb{R}^{2}$. Then,

$$
\|1-|v|\|_{L^{\infty}\left(\mathbb{R}^{2}\right)} \geq \frac{\varepsilon(v)^{2}}{10} .
$$

Moreover, there exists a universal constant $K_{2}>0$ such that

$$
\varepsilon(v) \leq K_{2} E(v) \text {. }
$$

In particular, the solutions $u_{\mathfrak{p}}$ given by Theorem 1 , satisfy in view of Proposition 1 ,

$$
\varepsilon_{\mathfrak{p}} \equiv \varepsilon\left(u_{\mathfrak{p}}\right) \rightarrow 0, \text { as } \mathfrak{p} \rightarrow 0,
$$

so that we deal with a transonic limit. In $[\mathbf{2 0 , 2 2 , 2 1 ]}$, it is proposed to study this transonic limit of solutions $v$ in the new anisotropic space scale,

$$
\tilde{x}_{1}=\varepsilon(v) x_{1}, \text { and } \tilde{x}_{2}=\frac{\varepsilon(v)^{2}}{\sqrt{2}} x_{2} .
$$

Considering the real-valued function

$$
\eta \equiv 1-|v|^{2}
$$


and performing the change of variables above, we introduce the rescaled map $N_{v}$ defined by

$$
N_{v}(x)=\frac{6}{\varepsilon(v)^{2}} \eta\left(\frac{x_{1}}{\varepsilon(v)}, \frac{\sqrt{2} x_{2}}{\varepsilon(v)^{2}}\right) .
$$

Notice that the same long-wave anisotropic scaling is performed to derive the Kadomtsev-Petviashvili equation, for instance in the water-wave context (see e.g.

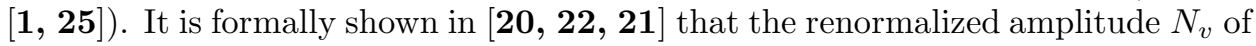
solutions to (TWc) converges, as the speed $c(v)$ converges to $\sqrt{2}$, i.e. as $\varepsilon(v) \rightarrow 0$, to solitary wave solutions to the two-dimensional Kadomtsev-Petviashvili equation (KP I), that is

$$
\partial_{t} \psi+\psi \partial_{1} \psi+\partial_{1}^{3} \psi-\partial_{1}^{-1}\left(\partial_{2}^{2} \psi\right)=0
$$

Our main goal in this paper is to provide a rigorous mathematical proof of that convergence for the branch of minimizing solutions presented in Theorem 1.

Solitary waves are localized solutions to (KP I) of the form $\psi(x, t)=w\left(x_{1}-\right.$ $\left.\sigma t, x_{2}\right)$, where $w$ belongs to the energy space for (KP I), i.e. the space $Y\left(\mathbb{R}^{2}\right)$ defined as the closure of $\partial_{1} \mathcal{C}_{c}^{\infty}\left(\mathbb{R}^{2}\right)$ for the norm

$$
\left\|\partial_{1} f\right\|_{Y\left(\mathbb{R}^{2}\right)} \equiv\left(\|\nabla f\|_{L^{2}\left(\mathbb{R}^{2}\right)}^{2}+\left\|\partial_{1}^{2} f\right\|_{L^{2}\left(\mathbb{R}^{2}\right)}^{2}\right)^{\frac{1}{2}} .
$$

The parameter $\sigma \geq 0$ denotes the speed of the solitary wave. The equation of a solitary wave $w$ of speed $\sigma=1$ is given by

$$
\partial_{1} w-w \partial_{1} w-\partial_{1}^{3} w+\partial_{1}^{-1}\left(\partial_{2}^{2} w\right)=0
$$

When $w \in Y\left(\mathbb{R}^{2}\right)$, the function $\partial_{1}^{-1} \partial_{2} w$ is well-defined (see [10]), so that (SW) makes sense.

In contrast with the Gross-Pitaevskii equation, the range of speeds is the full positive axis. In particular, there are no solitary waves of negative speed (see [10]). Given any $\sigma \geq 0$, a solitary wave $w_{\sigma}$ of speed $\sigma$ is deduced from a solution $w$ to (SW) by the scaling

$$
w_{\sigma}\left(x_{1}, x_{2}\right)=\sigma w\left(\sqrt{\sigma} x_{1}, \sigma x_{2}\right) .
$$

Solitary waves may be obtained in dimension two minimizing the Hamiltonian keeping the $L^{2}$-norm fixed (see $[\mathbf{9}, \mathbf{1 0}]$ ). Like (GP), equation (KP I) is indeed hamiltonian, with Hamiltonian given by

$$
E_{K P}(\psi)=\frac{1}{2} \int_{\mathbb{R}^{2}}\left(\partial_{1} \psi\right)^{2}+\frac{1}{2} \int_{\mathbb{R}^{2}}\left(\partial_{1}^{-1}\left(\partial_{2} \psi\right)\right)^{2}-\frac{1}{6} \int_{\mathbb{R}^{2}} \psi^{3},
$$

and the $L^{2}$-norm of $\psi$ is conserved as well. Setting

$$
S(N)=E_{K P}(N)+\frac{\sigma}{2} \int_{\mathbb{R}^{2}} N^{2}
$$

we term ground state, a solitary wave $N$ which minimizes the action $S$ among all non-constant solitary waves of speed $\sigma$ (see [11] for more details). In dimension two, a solitary wave is a ground state if and only if it minimizes the Hamiltonian $E_{K P}$ keeping the $L^{2}$-norm fixed (see $[\mathbf{9}]$ ). The constant $\mathcal{S}_{K P}$, which appears in Theorem 1, denotes the action $S(N)$ of the ground states $N$ of speed $\sigma=1$. 
Going back to the solutions $u_{\mathfrak{p}}$ of Theorem 1, we may drop the invariance under translations of our problem, assuming without loss of generality, since $\left|u_{\mathfrak{p}}(x)\right| \rightarrow 1$, as $|x| \rightarrow+\infty$ (see $[\mathbf{1 4}]$ ), that $\eta_{\mathfrak{p}} \equiv 1-\left|u_{\mathfrak{p}}\right|^{2}$ achieves its maximum at the origin, i.e.

$$
\left\|\eta_{\mathfrak{p}}\right\|_{L^{\infty}\left(\mathbb{R}^{2}\right)}=\left|\eta_{\mathfrak{p}}(0)\right| \text {. }
$$

We next consider the map

$$
N_{\mathfrak{p}} \equiv N_{u_{\mathfrak{p}}}
$$

Notice that the origin is a maximum point for $N_{\mathfrak{p}}$, and that in view of $(5)$, we have

$$
N_{\mathfrak{p}}(0) \geq \frac{3}{5}
$$

Our main result is

TheOREM 2. There exists a subsequence $\left(\mathfrak{p}_{n}\right)_{n \in \mathbb{N}}$, tending to 0 as $n$ tends to $+\infty$, and a ground state $N_{0}$ of (KP I) such that

$$
N_{\mathfrak{p}_{n}} \rightarrow N_{0} \text { in } W^{k, q}\left(\mathbb{R}^{2}\right) \text {, as } n \rightarrow+\infty,
$$

for any $k \in \mathbb{N}$ and any $1<q \leq+\infty$.

REMARK 1. There is a well-known explicit solitary wave solution to (KP I) of speed 1, namely the so-called "lump" solution, which may be written as

$$
w_{\ell}\left(x_{1}, x_{2}\right)=24 \frac{3-x_{1}^{2}+x_{2}^{2}}{\left(3+x_{1}^{2}+x_{2}^{2}\right)^{2}} \text {. }
$$

It is conjectured that the "lump" solution is a ground state. It is also conjectured that the ground state is unique, up to the invariances of the problem. If this was the case, then the full family $\left(N_{\mathfrak{p}}\right)_{\mathfrak{p}>0}$ would converge to $w_{\ell}$, as $\mathfrak{p} \rightarrow 0$.

So far, we have only discussed properties of the modulus of $u_{\mathfrak{p}}$. However, in our argument, the phase is central as well. More precisely, if $\mathfrak{p}$ is sufficiently small, then $u_{\mathfrak{p}}$ has no zero in view of (5), and we may lift it as $u_{\mathfrak{p}}=\varrho_{\mathfrak{p}} \exp i \varphi_{\mathfrak{p}}$. Setting

$$
\Theta_{\mathfrak{p}}(x)=\frac{6 \sqrt{2}}{\varepsilon_{\mathfrak{p}}} \varphi_{\mathfrak{p}}\left(\frac{x_{1}}{\varepsilon_{\mathfrak{p}}}, \frac{\sqrt{2} x_{2}}{\varepsilon_{\mathfrak{p}}^{2}}\right),
$$

we prove

Proposition 2. Let $\left(\mathfrak{p}_{n}\right)_{n \in \mathbb{N}}$ and $N_{0}$ be as in Theorem 2. Passing possibly to a further subsequence, we have

$$
\partial_{1} \Theta_{\mathfrak{p}_{n}} \rightarrow N_{0} \text { in } W^{k, q}\left(\mathbb{R}^{2}\right), \text { as } n \rightarrow+\infty,
$$

for any $k \in \mathbb{N}$ and any $1<q \leq+\infty$.

REMARK 2. Equation (KP I) is a higher dimensional extension of the wellknown Korteweg-de Vries equation $(\mathrm{KdV})$, which may be written as

$$
\partial_{t} \psi+\psi \partial_{1} \psi+\partial_{1}^{3} \psi=0
$$

In dimension one, travelling wave solutions $v_{c}$ to (TWc) are related to the classical soliton of the Korteweg-de Vries equation as follows. Setting $\varepsilon=\sqrt{2-c^{2}}$, we consider the rescaled function

$$
N_{\varepsilon}(x)=\frac{6}{\varepsilon^{2}} \eta_{c}\left(\frac{x}{\varepsilon}\right)
$$


where $\eta_{c} \equiv 1-\left|v_{c}\right|^{2}$. An explicit integration of (TWc) in dimension one leads to

$$
N_{\varepsilon}(x)=N(x) \equiv \frac{3}{\operatorname{ch}^{2}\left(\frac{x}{2}\right)},
$$

where $N$ is the classical soliton to the Korteweg-de-Vries equation. Concerning the phase $\varphi_{c}$ of $v_{c}$, we consider the scale change

$$
\Theta_{\varepsilon}(x)=\frac{6 \sqrt{2}}{\varepsilon} \varphi_{c}\left(\frac{x}{\varepsilon}\right)
$$

so that we obtain similarly

$$
\Theta_{\varepsilon}(x)^{\prime}=\sqrt{1-\frac{\varepsilon^{2}}{2}} \frac{N(x)}{1-\frac{\varepsilon^{2}}{2} N(x)} \rightarrow N(x), \text { as } \varepsilon \rightarrow 0 .
$$

REMARK 3. Let $u_{c}$ be a solution to (TWc) in dimension three, which may be written as $u_{c}=\varrho_{c} \exp i \varphi_{c}$, and denote

$$
N_{c}(x)=\frac{6}{\varepsilon^{2}} \eta_{c}\left(\frac{x_{1}}{\varepsilon}, \frac{\sqrt{2} x_{2}}{\varepsilon^{2}}, \frac{\sqrt{2} x_{3}}{\varepsilon^{2}}\right), \text { and } \Theta_{c}(x)=\frac{6 \sqrt{2}}{\varepsilon} \varphi_{c}\left(\frac{x_{1}}{\varepsilon}, \frac{\sqrt{2} x_{2}}{\varepsilon^{2}}, \frac{\sqrt{2} x_{3}}{\varepsilon^{2}}\right),
$$

where $\eta_{c} \equiv 1-\varrho_{c}^{2}$ and $\varepsilon=\sqrt{2-c^{2}}$. Then, it is also formally shown in $[\mathbf{2 0 , 2 2 , \mathbf { 2 1 } ]}$ that the functions $N_{c}$ and $\partial_{1} \Theta_{c}$ converge, as the parameter $\varepsilon$ converges to 0 , to a solitary wave solution $w$ to the three-dimensional Kadomtsev-Petviashvili equation (KP I), which writes

$$
\partial_{t} \psi+\psi \partial_{1} \psi+\partial_{1}^{3} \psi-\partial_{1}^{-1}\left(\partial_{2}^{2} \psi+\partial_{3}^{2} \psi\right)=0
$$

In particular, the equation for the solitary wave $w$ is now written as

$$
\partial_{1} w-w \partial_{1} w-\partial_{1}^{3} w+\partial_{1}^{-1}\left(\partial_{2}^{2} w+\partial_{3}^{2} w\right)=0 .
$$

However, the existence of a transonic branch of solutions is still an open problem, at least on the mathematical level. This branch of solutions is conjectured in $[\mathbf{2 0 , 2 2}]$ in view of numerical computations and formal arguments.

1.2. Some elements in the proofs. The first element in the proofs of Theorem 2 and Proposition 2 deals with the asymptotic behaviour of $\varepsilon_{\mathfrak{p}}$ as a function of $\mathfrak{p}$.

LEMMA $1([\mathbf{3}])$. Let $\varepsilon_{\mathfrak{p}}=\varepsilon\left(u_{\mathfrak{p}}\right)=\sqrt{2-c\left(u_{\mathfrak{p}}\right)^{2}}$. There exist some positive constants $K_{3}$ and $K_{4}$, not depending on $\mathfrak{p}$, such that

$$
K_{3} \mathfrak{p} \leq \varepsilon_{\mathfrak{p}} \leq K_{4} \mathfrak{p}
$$

for any $\mathfrak{p}$ sufficiently small.

The second step is to derive estimates on the renormalized maps $N_{\mathfrak{p}}$, which do not depend on $\mathfrak{p}$. More precisely, we prove

Proposition 3. Let $k \in \mathbb{N}$ and $1<q \leq+\infty$. There exists some constant $K(k, q)$, depending possibly on $k$ and $q$, but not on $\mathfrak{p}$, such that

$$
\left\|N_{\mathfrak{p}}\right\|_{W^{k, q}\left(\mathbb{R}^{2}\right)}+\left\|\partial_{1} \Theta_{\mathfrak{p}}\right\|_{W^{k, q}\left(\mathbb{R}^{2}\right)}+\varepsilon_{\mathfrak{p}}\left\|\partial_{2} \Theta_{\mathfrak{p}}\right\|_{W^{k, q}\left(\mathbb{R}^{2}\right)} \leq K(k, q),
$$

for any $\mathfrak{p}$ sufficiently small. 
At this stage, we may invoke standard compactness theorems to assert that there exists some subsequence $\left(\mathfrak{p}_{n}\right)_{n \in \mathbb{N}}$, tending to 0 as $n$ tends to $+\infty$, and a function $N_{0}$ such that, for any $k \in \mathbb{N}$ and any compact subset $K$ of $\mathbb{R}^{2}$,

$$
N_{\mathfrak{p}_{n}} \rightarrow N_{0} \text { in } \mathcal{C}^{k}(K) \text {, as } n \rightarrow+\infty \text {. }
$$

In view of (8), we have

$$
N_{0}(0) \geq \frac{3}{5}
$$

so that $N_{0}$ is not identically constant. Moreover, we also have

LEMma 2. The function $N_{0}$ is a non-constant solution to (SW).

In order to complete the proof of Theorem 2, it remains to establish strong convergence on the whole plane. For this last step, we essentially rely on a variational argument, proving a kind of gamma-convergence of the energies, combined with a concentration-compactness result for constrained minimizers of (KP I) established in $[\mathbf{9}]$.

As a matter of fact, considering scalings (6) and (9), the momentum $p\left(u_{\mathfrak{p}}\right)$ can be expressed as

$$
p\left(u_{\mathfrak{p}}\right)=\frac{\varepsilon_{\mathfrak{p}}}{72} \int_{\mathbb{R}^{2}} N_{\mathfrak{p}} \partial_{1} \Theta_{\mathfrak{p}}
$$

while the energy $E\left(u_{\mathfrak{p}}\right)$ has the expansion

$$
E\left(u_{\mathfrak{p}}\right)=\sqrt{2} \frac{\varepsilon_{\mathfrak{p}}}{144}\left(E_{0}\left(N_{\mathfrak{p}}, \Theta_{\mathfrak{p}}\right)+\varepsilon_{\mathfrak{p}}^{2} E_{2}\left(N_{\mathfrak{p}}, \Theta_{\mathfrak{p}}\right)+\varepsilon_{\mathfrak{p}}^{4} E_{4}\left(N_{\mathfrak{p}}, \Theta_{\mathfrak{p}}\right)\right)
$$

It turns out that the functions $E_{0}, E_{2}$ and $E_{4}$ are uniformly bounded for $\mathfrak{p}$ approaching 0 . Moreover, $E_{0}$ and $E_{2}$ are given by the expressions

$$
E_{0}\left(N_{\mathfrak{p}}, \Theta_{\mathfrak{p}}\right)=\int_{\mathbb{R}^{2}}\left(N_{\mathfrak{p}}^{2}+\left(\partial_{1} \Theta_{\mathfrak{p}}\right)^{2}\right)
$$

and

$$
E_{2}\left(N_{\mathfrak{p}}, \Theta_{\mathfrak{p}}\right)=\int_{\mathbb{R}^{2}}\left(\frac{1}{2}\left(\partial_{1} N_{\mathfrak{p}}\right)^{2}+\frac{1}{2}\left(\partial_{2} \Theta_{\mathfrak{p}}\right)^{2}-\frac{1}{6} N_{\mathfrak{p}}\left(\partial_{1} \Theta_{\mathfrak{p}}\right)^{2}\right)
$$

In the course of our proof, we will show that

$$
N_{\mathfrak{p}} \sim \partial_{1} \Theta_{\mathfrak{p}}, \text { as } \mathfrak{p} \rightarrow 0,
$$

and that the difference is actually of order $\varepsilon_{\mathfrak{p}}^{2}$. This yields, at least heuristically,

$$
p\left(u_{\mathfrak{p}}\right) \sim \frac{\varepsilon_{\mathfrak{p}}}{72} \int_{\mathbb{R}^{2}} N_{\mathfrak{p}}^{2}, \text { and } E\left(u_{\mathfrak{p}}\right) \sim \sqrt{2} \frac{\varepsilon_{\mathfrak{p}}}{72} \int_{\mathbb{R}^{2}} N_{\mathfrak{p}}^{2} \sim \sqrt{2} p\left(u_{\mathfrak{p}}\right),
$$

so that the discrepancy term

$$
\Sigma\left(u_{\mathfrak{p}}\right)=\sqrt{2} p\left(u_{\mathfrak{p}}\right)-E\left(u_{\mathfrak{p}}\right),
$$

tends to 0 as $\mathfrak{p} \rightarrow+\infty$.

The (KP I) energy appears when we consider the second order term. Inserting at least formally relation (13) into (12), we are led to

$$
E_{2}\left(N_{\mathfrak{p}}, \Theta_{\mathfrak{p}}\right) \sim E_{K P}\left(N_{\mathfrak{p}}\right), \text { as } \mathfrak{p} \rightarrow 0 .
$$

Using some precise estimates on the solutions, we will actually show that

$$
E_{2}\left(N_{\mathfrak{p}}, \Theta_{\mathfrak{p}}\right) \sim E_{K P}\left(\partial_{1} \Theta_{\mathfrak{p}}\right), \text { as } \mathfrak{p} \rightarrow 0,
$$


since it turns out that it is easier to work, in view of the nonlocal term in the (KP I) energy, with $\partial_{1} \Theta_{\mathfrak{p}}$ than with $N_{\mathfrak{p}}$, these two terms having the same limit in view of (13).

The proof of (15) amounts to a careful analysis of any lower order terms, including terms provided by $E_{0}$. In particular, we obtain for the discrepancy functional,

LEMMA 3. We have

$$
\Sigma\left(u_{\mathfrak{p}}\right)=-\frac{\sqrt{2} \varepsilon_{\mathfrak{p}}^{3}}{144} E_{K P}\left(\partial_{1} \Theta_{\mathfrak{p}}\right)+\underset{\mathfrak{p} \rightarrow 0}{o}\left(\varepsilon_{\mathfrak{p}}^{3}\right)
$$

We then use the lower bound on $\Sigma\left(u_{\mathfrak{p}}\right)$ provided by the left-hand side of $(3)$ to derive a precise upper bound on $E_{K P}\left(\partial_{1} \Theta_{\mathfrak{p}}\right)$. More precisely, we show

LEMMA 4. We have

$$
-\frac{1}{54 \mathcal{S}_{K P}^{2}}\left(\int_{\mathbb{R}^{2}}\left(\partial_{1} \Theta_{\mathfrak{p}}\right)^{2}\right)^{3} \leq E_{K P}\left(\partial_{1} \Theta_{\mathfrak{p}}\right) \leq-\frac{1}{54 \mathcal{S}_{K P}^{2}}\left(\int_{\mathbb{R}^{2}}\left(\partial_{1} \Theta_{\mathfrak{p}}\right)^{2}\right)^{3}+\underset{\mathfrak{p} \rightarrow 0}{o}(1) \text {. }
$$

In particular, the function $\partial_{1} \Theta_{\mathfrak{p}}$, or alternatively $N_{\mathfrak{p}}$, has approximatively the energy of a ground state for (KP I) corresponding to its $L^{2}$-norm. The proof of Theorem 2 is then completed using a concentration-compactness argument of $[\mathbf{9}]$. This result yields the strong convergence of some subsequence $\left(\partial_{1} \Theta_{\mathfrak{p}_{n}}\right)_{n \in \mathbb{N}}$ in the space $Y\left(\mathbb{R}^{2}\right)$.

Proposition 4. There exists a subsequence $\left(\mathfrak{p}_{n}\right)_{n \in \mathbb{N}}$, tending to 0 as $n$ tends to $+\infty$, and a ground state $N_{0}$ of (KP I) such that

$$
\partial_{1} \Theta_{\mathfrak{p}_{n}} \rightarrow N_{0} \text { in } Y\left(\mathbb{R}^{2}\right) \text {, and } N_{\mathfrak{p}_{n}} \rightarrow N_{0} \text { in } L^{2}\left(\mathbb{R}^{2}\right) \text {, as } n \rightarrow+\infty \text {. }
$$

In order to improve the convergence, we finally invoke the estimates of Proposition 3. This concludes the proofs of Theorem 2 and Proposition 2 giving the convergence in any space $W^{k, q}\left(\mathbb{R}^{2}\right)$ by standard interpolation theory.

To conclude this introduction, let us emphasize that the results in this paper only concern travelling waves. This raises quite naturally the corresponding issue for the time-dependent equations. More precisely, in which sense do the Korteweg-de Vries equation in dimension one and the Kadomtsev-Petviashvili equation in higher dimensions approximate the Gross-Pitaevskii equation in the transonic limit ? Notice that this question has already been formally addressed in the one-dimensional case in $[\mathbf{2 4}]$.

1.3. Outline of the paper. The paper is organized as follows. Sections 2 and 3 are devoted to various properties of solitary wave solutions to (KP I) and travelling wave solutions to (TWc) which are subsequently used. In Section 4, we perform the expansion of ( $\mathrm{TWc}$ ) with respect to the small parameter $\varepsilon$ occurring in the definition of the slow space variables. Terms in this expansion are more clearly analyzed in Fourier variables. Various kernels then appear, which are studied in Section 5. In Section 6, we provide Sobolev bounds on $N_{\mathfrak{p}}$ and prove Proposition 3. Finally, we prove our main theorems in Section 7.

\section{Some properties of solitary wave solutions to (KP I)}

We first recall some facts about equation (KP I), which will enter in some places in our proofs. 
2.1. Rewriting the solitary wave equation. The existence and qualitative properties of the solutions $w$ to $(\mathrm{SW})$ in the energy space $Y\left(\mathbb{R}^{2}\right)$ are considered in the series of papers $[\mathbf{1 0}, \mathbf{1 1}, \mathbf{9}]$. In $[\mathbf{1 1}]$, a new formulation of $(\mathrm{SW})$ is provided which turns out be also fruitful in our context. Applying the operator $\partial_{1}$ to (SW), we obtain

$$
\partial_{1}^{4} w-\Delta w+\frac{1}{2} \partial_{1}^{2}\left(w^{2}\right)=0 .
$$

The Fourier transform of (2.1) has the following simple form

$$
\widehat{w}(\xi)=\frac{1}{2} \frac{\xi_{1}^{2}}{|\xi|^{2}+\xi_{1}^{4}} \widehat{w^{2}}(\xi)
$$

so that we may recast (2.1) as a convolution equation

$$
w=\frac{1}{2} K_{0} \star w^{2}
$$

where the Fourier transform of the kernel $K_{0}$ is given by

$$
\widehat{K_{0}}(\xi)=\frac{\xi_{1}^{2}}{|\xi|^{2}+\xi_{1}^{4}}
$$

In view of (2.2), equation (2.3) provides an equivalent formulation to (SW), i.e. any solution $w$ to $(2.3)$ in the energy space $Y\left(\mathbb{R}^{2}\right)$ is also solution to $(\mathrm{SW})$.

Several properties of the kernel $K_{0}$ are studied in [18]. In particular, it is proved there that $K_{0}$ belongs to $L^{p}\left(\mathbb{R}^{2}\right)$ for any $1<p<3$ (see also Lemma 5.1).

2.2. Existence of ground state solutions. Given any $\mu \geq 0$, the minimization problem

$$
\left(\mathcal{P}_{K P}(\mu)\right) \quad \mathcal{E}_{\min }^{K P}(\mu)=\inf \left\{E_{K P}(w), w \in Y\left(\mathbb{R}^{2}\right), \int_{\mathbb{R}^{2}}|w|^{2}=\mu\right\},
$$

is considered in $[\mathbf{9}]$, where the existence of minimizers is established. The minimizers $N$ for this problem happen to be ground states for (KP I). They are solutions to

$$
\sigma \partial_{1} N-N \partial_{1} N-\partial_{1}^{3} N+\partial_{1}^{-1}\left(\partial_{2}^{2} N\right)=0
$$

The speed $\sigma$ appears as a Lagrange multiplier associated to $\left(\mathcal{P}_{K P}(\mu)\right)$. In particular, $\sigma$ is not necessarily equal to 1 . The proof in $[\mathbf{9}]$ relies on the following concentration-compactness result, which gives the compactness of minimizing sequences to $\left(\mathcal{P}_{K P}(\mu)\right)$.

THEOREM $2.1([\mathbf{9}])$. Let $\mu \geq 0$, and let $\left(w_{n}\right)_{n \in \mathbb{N}}$ be a minimizing sequence to $\left(\mathcal{P}_{K P}(\mu)\right)$ in $Y\left(\mathbb{R}^{2}\right)$. Then, there exist some points $\left(a_{n}\right)_{n \in \mathbb{N}}$ and a function $N \in Y\left(\mathbb{R}^{2}\right)$ such that, up to some subsequence,

$$
w_{n}\left(\cdot-a_{n}\right) \rightarrow N \text { in } Y\left(\mathbb{R}^{2}\right) \text {, as } n \rightarrow+\infty .
$$

The limit function $N$ is solution to the minimization problem $\left(\mathcal{P}_{K P}(\mu)\right)$. In particular, $N$ is a ground state for (KP I). 
2.3. Scale invariance. As mentioned in the introduction, if $w$ is solution to (SW), then, for any $\sigma>0$, the map $w_{\sigma}$ defined by $(7)$ is solution to $(2.5)$, i.e. $w_{\sigma}$ is a solitary wave solution to (KP I) with speed $\sigma$. Concerning the energy, we notice that

$$
\int_{\mathbb{R}^{2}}\left|w_{\sigma}\right|^{2}=\sqrt{\sigma} \int_{\mathbb{R}^{2}}|w|^{2}, \int_{\mathbb{R}^{2}}\left|w_{\sigma}\right|^{3}=\sigma^{\frac{3}{2}} \int_{\mathbb{R}^{2}}|w|^{3}, \int_{\mathbb{R}^{2}}\left|\partial_{1} w_{\sigma}\right|^{2}=\sigma^{\frac{3}{2}} \int_{\mathbb{R}^{2}}\left|\partial_{1} w\right|^{2},
$$

and

$$
\int_{\mathbb{R}^{2}}\left(\partial_{1}^{-1}\left(\partial_{2} w_{\sigma}\right)\right)^{2}=\sigma^{\frac{3}{2}} \int_{\mathbb{R}^{2}}\left(\partial_{1}^{-1}\left(\partial_{2} w\right)\right)^{2}
$$

It follows that

$$
E_{K P}\left(w_{\sigma}\right)=\sigma^{\frac{3}{2}} E_{K P}(w), \text { and } \int_{\mathbb{R}^{2}}\left|w_{\sigma}\right|^{2}=\sqrt{\sigma} \int_{\mathbb{R}^{2}}|w|^{2}
$$

It is shown in $[\mathbf{9}]$ that ground states $N$ with speed $\sigma=1$ correspond to solutions to $\left(\mathcal{P}_{K P}(\mu)\right)$ for

$$
\mu=\mu^{*} \equiv 3 \mathcal{S}_{K P} .
$$

As a matter of fact, it is proved in $[\mathbf{1 0}, \mathbf{1 8}]$ that any solution $w$ to $(\mathrm{SW})$ satisfies the relations

$$
E_{K P}(w)=-\frac{1}{6} \int_{\mathbb{R}^{2}} w^{2}, \text { and } S(w)=\frac{1}{3} \int_{\mathbb{R}^{2}} w^{2},
$$

so that the energy and the $L^{2}$-norm of ground states $N$ with speed $\sigma=1$ are given by

$$
E_{K P}(N)=-\frac{1}{2} \mathcal{S}_{K P}, \text { and } \int_{\mathbb{R}^{2}} N^{2}=3 \mathcal{S}_{K P}=\mu^{*}
$$

Relations (2.6) then provide

Lemma 2.1. Let $N \in Y\left(\mathbb{R}^{2}\right)$. Given any $\sigma \geq 0$, the map $N_{\sigma}$ defined by (7) is a minimizer for $\mathcal{E}_{\min }^{K P}\left(\sqrt{\sigma} \mu^{*}\right)$ if and only if $N$ is a minimizer for $\mathcal{E}_{\min }^{K P}\left(\mu^{*}\right)$. In particular, we have

$$
\mathcal{E}_{\min }^{K P}(\mu)=-\frac{\mu^{3}}{54 \mathcal{S}_{K P}^{2}}, \forall \mu \geq 0 .
$$

Moreover, $N_{\sigma}$ and $N$ are ground states for (KP I), with speed $\sigma$, respectively, 1 . In particular, they are solutions to (2.5), respectively, (SW).

Proof. Given any $\mu>0$, we denote $\Lambda_{\mu}^{2}\left(\mathbb{R}^{2}\right)=\left\{w \in L^{2}\left(\mathbb{R}^{2}\right)\right.$, s.t. $\int_{\mathbb{R}^{2}}|w|^{2}=$ $\mu\}$. In view of $(2.6)$, the function $w \mapsto w_{\sigma}$ maps $\Lambda_{\mu^{*}}^{2}\left(\mathbb{R}^{2}\right)$ onto $\Lambda_{\mu^{*} \sqrt{\sigma}}^{2}\left(\mathbb{R}^{2}\right)$, such that

$$
E_{K P}\left(w_{\sigma}\right)=\sigma^{\frac{3}{2}} E_{K P}(w) .
$$

Hence, $N_{\sigma}$ is a minimizer for $\mathcal{E}_{\min }^{K P}\left(\mu^{*} \sqrt{\sigma}\right)$ if and only if $N$ is a minimizer for $\mathcal{E}_{\min }^{K P}\left(\mu^{*}\right)$. Moreover,

$$
\mathcal{E}_{\min }^{K P}\left(\mu^{*} \sqrt{\sigma}\right)=\sigma^{\frac{3}{2}} \mathcal{E}_{\min }^{K P}\left(\mu^{*}\right)=-\frac{\sigma^{\frac{3}{2}} \mathcal{S}_{K P}}{2} .
$$

Identity $(2.7)$ follows letting $\sigma=\frac{\mu^{2}}{\left(\mu^{*}\right)^{2}}$. The last statements of Lemma 2.1 are proved in $[\mathbf{9}]$. 
In the course of our proofs, we will encounter sequences $\left(w_{n}\right)_{n \in \mathbb{N}}$ which are not exactly minimizing sequences for $\left(\mathcal{P}_{K P}(\mu)\right)$, but which satisfy

$$
E_{K P}\left(w_{n}\right) \rightarrow \mathcal{E}_{\min }^{K P}(\mu) \text {, and } \int_{\mathbb{R}^{2}} w_{n}^{2} \rightarrow \mu \text {, as } n \rightarrow+\infty,
$$

for some positive number $\mu$. In this case, we will invoke the following variant (and in fact, consequence) of Theorem 2.1.

Proposition 2.1. Let $\mu_{0}>0$, and $\left(w_{n}\right)_{n \in \mathbb{N}}$ denote a sequence of functions in $Y\left(\mathbb{R}^{2}\right)$ satisfying (2.8) for $\mu=\mu_{0}$. Then, there exist some points $\left(a_{n}\right)_{n \in \mathbb{N}}$ and a ground state solution $N_{\sigma}$ to $(2.5)$, with $\sigma=\frac{\mu_{0}^{2}}{\left(\mu^{*}\right)^{2}}$, such that, up to some subsequence,

$$
w_{n}\left(\cdot-a_{n}\right) \rightarrow N_{\sigma} \text { in } Y\left(\mathbb{R}^{2}\right), \text { as } n \rightarrow+\infty .
$$

Proof. We denote

$$
\mu_{n}=\int_{\mathbb{R}^{2}} w_{n}^{2}, \text { and } \sigma_{n}=\frac{\mu_{0}^{2}}{\mu_{n}^{2}},
$$

and consider the functions

$$
z_{n}\left(x_{1}, x_{2}\right)=\sigma_{n} w_{n}\left(\sqrt{\sigma_{n}} x_{1}, \sigma_{n} x_{2}\right)
$$

In view of (2.6) and (2.8),

$$
\sigma_{n} \rightarrow 1, \text { as } n \rightarrow+\infty
$$

and $\left(z_{n}\right)_{n \in \mathbb{N}}$ is a minimizing sequence of $\left(\mathcal{P}_{K P}(\mu)\right)$ for $\mu=\mu_{0}$. Therefore, by Theorem 2.1, there exist some points $\left(a_{n}\right)_{n \in \mathbb{N}}$ and a minimizer $N_{\sigma}$ to $\left(\mathcal{P}_{K P}(\mu)\right)$ for $\mu=\mu_{0}$ such that, up to some subsequence,

$$
z_{n}\left(\cdot-a_{n}\right) \rightarrow N_{\sigma} \text { in } Y\left(\mathbb{R}^{2}\right), \text { as } n \rightarrow+\infty .
$$

In particular, it follows from Lemma 2.1 that $N_{\sigma}$ is solution to $(2.5)$, with $\sigma=\frac{\mu_{0}^{2}}{\left(\mu^{*}\right)^{2}}$. We now denote

$$
N_{n}\left(x_{1}, x_{2}\right)=\frac{1}{\sigma_{n}} N_{\sigma}\left(\frac{x_{1}}{\sqrt{\sigma_{n}}}, \frac{x_{2}}{\sigma_{n}}\right),
$$

so that, by the change of variables $\left(y_{1}, y_{2}\right)=\left(\sqrt{\sigma_{n}} x_{1}, \sigma_{n} x_{2}\right)$,

$$
\begin{aligned}
\left\|z_{n}\left(\cdot-a_{n}\right)-N_{\sigma}\right\|_{Y\left(\mathbb{R}^{2}\right)}^{2}= & \sqrt{\sigma_{n}}\left\|w_{n}\left(\cdot-a_{n}\right)-N_{n}\right\|_{L^{2}\left(\mathbb{R}^{2}\right)}^{2} \\
& +\sigma_{n}^{\frac{3}{2}}\left\|\partial_{1} w_{n}\left(\cdot-a_{n}\right)-\partial_{1} N_{n}\right\|_{L^{2}\left(\mathbb{R}^{2}\right)}^{2} \\
+ & \sigma_{n}^{\frac{3}{2}}\left\|\partial_{1}^{-1} \partial_{2} w_{n}\left(\cdot-a_{n}\right)-\partial_{1}^{-1} \partial_{2} N_{n}\right\|_{L^{2}\left(\mathbb{R}^{2}\right)}^{2} .
\end{aligned}
$$

By (2.9) and (2.10), we have

$$
w_{n}\left(\cdot-a_{n}\right)-N_{n} \rightarrow 0 \text { in } Y\left(\mathbb{R}^{2}\right) \text {, as } n \rightarrow+\infty .
$$

Proposition 2.1 follows provided we first prove that

$$
N_{n} \rightarrow N_{\sigma} \text { in } Y\left(\mathbb{R}^{2}\right) \text {, as } n \rightarrow+\infty .
$$

This last assertion is itself a consequence of the general observation that

$$
\lambda \psi(\sqrt{\mu} \cdot \mu \cdot) \rightarrow \psi \text { in } L^{2}\left(\mathbb{R}^{2}\right), \text { as } \lambda \rightarrow 1 \text { and } \mu \rightarrow 1,
$$

which may be deduced from the dominated convergence theorem, when $\psi$ is in $\mathcal{C}_{c}^{\infty}\left(\mathbb{R}^{2}\right)$, then, using the density of $\mathcal{C}_{c}^{\infty}\left(\mathbb{R}^{2}\right)$ into $L^{2}\left(\mathbb{R}^{2}\right)$, when $\psi$ only belongs to $L^{2}\left(\mathbb{R}^{2}\right)$. 


\section{Some properties of solutions to (TWc)}

In this section, we gather a number of properties of solutions to (TWc), which enter in our asymptotic analysis. Most of these results are available in the literature on the subject.

3.1. General solutions. Let $v$ be a finite energy solution to (TWc) on $\mathbb{R}^{2}$. It can be shown using various elliptic estimates (see $[\mathbf{1 2}, \mathbf{2 8}, \mathbf{3}]$ ) that there exists some positive constant $K$, not depending on $c$, such that

$$
\|1-|v|\|_{L^{\infty}\left(\mathbb{R}^{2}\right)} \leq 1
$$

and

$$
\|\nabla v\|_{L^{\infty}\left(\mathbb{R}^{2}\right)} \leq K\left(1+\frac{c^{2}}{4}\right)^{\frac{3}{2}} .
$$

In view of (4), estimates (3.1) and (3.2) may be recast as

$$
\|\eta\|_{L^{\infty}\left(\mathbb{R}^{2}\right)}+\|\nabla v\|_{L^{\infty}\left(\mathbb{R}^{2}\right)} \leq K
$$

where we have set $\eta \equiv 1-|v|^{2}$. For higher order derivatives, it similarly follows from the proof of Lemma 2.1 in $[\mathbf{3}]$ that there exists some positive constant $K(k)$, not depending on $c$, such that

$$
\|v\|_{\mathcal{C}^{k}\left(\mathbb{R}^{2}\right)} \leq K(k)
$$

for any $k \in \mathbb{N}$.

More generally, we have

$$
\|\eta\|_{W^{k, q}\left(\mathbb{R}^{2}\right)}+\|\nabla v\|_{W^{k, q}\left(\mathbb{R}^{2}\right)} \leq K(c, k, q),
$$

for any $k \in \mathbb{N}$ and any $1<q<+\infty$ (see [16]). Notice that the constant $K(c, k, q)$ possibly depends on the speed $c$, so that we may have

$$
K(c, k, q) \rightarrow+\infty, \text { as } c \rightarrow \sqrt{2} .
$$

Before establishing the convergence of the rescaled functions $N_{\mathfrak{p}}$ and $\Theta_{\mathfrak{p}}$, we shall need to establish their boundedness in the spaces $W^{k, q}\left(\mathbb{R}^{2}\right)$. This requires to get some control upon the dependence on $c$ of the constant $K(c, k, q)$. The proof of Proposition 3 in Section 6 below provides such a control.

We will also take advantage of the fact that the maps $u_{\mathfrak{p}}$ have small energy. Indeed, in view of (4) and elliptic estimate (3.3), we may show that, if a solution $v$ to (TWc) has sufficiently small energy, it does not vanish. More precisely, we have

LEmma $3.1([\mathbf{3}])$. There exists a universal constant $E_{0}$ such that, if $v$ is a solution to (TWc) which satisfies $E(v) \leq E_{0}$, then

$$
\frac{1}{2} \leq|v| \leq 2 .
$$

If $v$ satisfies (3.6), then we may lift it as

$$
v=\varrho \exp i \varphi,
$$

where $\varphi$ is a real-valued, smooth function on $\mathbb{R}^{2}$ defined modulo a multiple of $2 \pi$. We have in that case,

$$
\partial_{j} v=\left(i \varrho \partial_{j} \varphi+\partial_{j} \varrho\right) \exp i \varphi,
$$


so that

$$
\left\langle i \partial_{1} v, v\right\rangle=-\varrho^{2} \partial_{1} \varphi, \text { and } e(v)=\frac{1}{2}\left(|\nabla \varrho|^{2}+\varrho^{2}|\nabla \varphi|^{2}\right)+\frac{1}{4} \eta^{2} .
$$

Moreover, the momentum $p$ takes the simple form

$$
p(v)=\frac{1}{2} \int_{\mathbb{R}^{2}} \eta \partial_{1} \varphi
$$

The system of equations for $\varrho$ and $\varphi$ is written as

$$
\frac{c}{2} \partial_{1} \varrho^{2}+\operatorname{div}\left(\varrho^{2} \nabla \varphi\right)=0,
$$

and

$$
c \varrho \partial_{1} \varphi-\Delta \varrho-\varrho\left(1-\varrho^{2}\right)+\varrho|\nabla \varphi|^{2}=0 .
$$

Combining both the equations, the quantity $\eta$ satisfies

$$
\Delta^{2} \eta-2 \Delta \eta+c^{2} \partial_{1}^{2} \eta=-2 \Delta\left(|\nabla v|^{2}+\eta^{2}-c \eta \partial_{1} \varphi\right)-2 c \partial_{1} \operatorname{div}(\eta \nabla \varphi),
$$

where the left-hand side is linear with respect to $\eta$, whereas the right-hand side is (almost) quadratic with respect to $\eta$ and $\nabla \varphi$.

Multiplying (3.8) by $\varphi$ and integrating by parts, we obtain a first relation for the momentum

$$
c p(v)=\int_{\mathbb{R}^{N}} \varrho^{2}|\nabla \varphi|^{2} .
$$

In another direction, Pohozaev identities yield

$$
E(v)=\int_{\mathbb{R}^{2}}\left|\partial_{1} v\right|^{2}, \text { and } E(v)=\int_{\mathbb{R}^{2}}\left|\partial_{2} v\right|^{2}+c p(v) .
$$

Introducing the quantities $\Sigma(v)=\sqrt{2} p(v)-E(v)$, the second identity in (3.11) may be recast as

$$
\int_{\mathbb{R}^{2}}\left|\partial_{2} v\right|^{2}+\Sigma(v)=\left(\sqrt{2}-\sqrt{2-\varepsilon(v)^{2}}\right) p(v)=\frac{\varepsilon(v)^{2}}{\sqrt{2}+\sqrt{2-\varepsilon(v)^{2}}} p(v) .
$$

In the case $\Sigma(v)>0$, this yields an interesting estimate for the transversal derivative $\partial_{2} v$. Adding both the equalities in (3.11), we also derive a second relation for the momentum

$$
\frac{1}{2} \int_{\mathbb{R}^{2}} \eta^{2}=c p(v) .
$$

With similar arguments and combining with (3.10), we are led to

LEMMA $3.2([\mathbf{3}])$. Let $v$ be a finite energy solution to $(\mathrm{TWc})$ on $\mathbb{R}^{2}$ satisfying (3.6). Then, we have the identities

$$
\begin{gathered}
\Sigma(v)+\frac{1}{2} \int_{\mathbb{R}^{2}}|\nabla \varrho|^{2}=\frac{\varepsilon(v)^{2}}{\sqrt{2}+c(v)} p(v), \\
\int_{\mathbb{R}^{2}}|\nabla \varrho|^{2}\left(1+\frac{1}{\varrho^{2}}\right)=\int_{\mathbb{R}^{2}} \eta|\nabla \varphi|^{2},
\end{gathered}
$$

and the inequality

$$
E(v) \leq 7 c(v)^{2} \int_{\mathbb{R}^{2}} \eta^{2}
$$


In view of definition (1), we have

$$
\int_{\mathbb{R}^{2}} \eta^{2} \leq 4 E(v)
$$

so that inequality (3.15) shows that the energy is comparable to the integral of $\eta^{2}$ for any solutions $v$ satisfying (3.6). When $\Sigma(v)>0$, identity (3.13) shows that

$$
\Sigma(v) \leq \frac{\varepsilon(v)^{2}}{\sqrt{2}} p(v) \leq K E(v)^{2} p(v) \leq 2 K p(v)^{3},
$$

where we have invoked Proposition 1 for the second inequality. In particular, we obtain

$$
E(v) \sim \sqrt{2} p(v)
$$

as $E(v)$, or $p(v)$, approaches 0 .

In several places (in particular, in the proof of Proposition 3), we shall need estimates for higher order derivatives. For that purpose, we shall use

LEMMA 3.3. Let $1<q<+\infty$, and let $v$ be a finite energy solution to (TWc) on $\mathbb{R}^{2}$ satisfying (3.6). Then, there exists some constant $K(q)$, not depending on c, such that

$$
\|\nabla \varphi\|_{L^{q}\left(\mathbb{R}^{2}\right)} \leq K(q)\|\eta\|_{L^{q}\left(\mathbb{R}^{2}\right)},
$$

More generally, given any index $\alpha=\left(\alpha_{1}, \alpha_{2}\right) \in \mathbb{N}^{2}$, there exist some constants $K(q, \alpha)$, not depending on $c$, such that

$$
\left\|\partial^{\alpha}(\nabla \varphi)\right\|_{L^{q}\left(\mathbb{R}^{2}\right)} \leq K(q, \alpha)\left(\left\|\partial^{\alpha} \eta\right\|_{L^{q}\left(\mathbb{R}^{2}\right)}+\sum_{0 \leq \beta<\alpha}\left\|\partial^{\beta} \eta\right\|_{L^{\infty}\left(\mathbb{R}^{2}\right)}\left\|\partial^{\alpha-\beta}(\nabla \varphi)\right\|_{L^{q}\left(\mathbb{R}^{2}\right)}\right) .
$$

Proof. First notice that in view of (3.4) and (3.5), the functions $\eta$ and $\nabla \varphi$ belong to $W^{k, q}\left(\mathbb{R}^{2}\right)$ for any $k \in \mathbb{N}$ and any $1<q \leq+\infty$. In particular, the norms in inequalities (3.16) and (3.17) are well-defined and finite. Lemma 3.3 is then a consequence of the elliptic nature of equation (3.8), which may be written as

$$
\Delta \varphi=\frac{c}{2} \partial_{1} \eta+\operatorname{div}(\eta \nabla \varphi)
$$

so that, more generally,

$$
\Delta\left(\partial^{\alpha} \varphi\right)=\frac{c}{2} \partial_{1} \partial^{\alpha} \eta+\operatorname{div}\left(\partial^{\alpha}(\eta \nabla \varphi)\right)
$$

for any $\alpha \in \mathbb{N}^{2}$. Using standard elliptic estimates and inequality (4), we derive from (3.18) that

$$
\left\|\nabla\left(\partial^{\alpha} \varphi\right)\right\|_{L^{q}\left(\mathbb{R}^{2}\right)} \leq K(q)\left(\left\|\partial^{\alpha} \eta\right\|_{L^{q}\left(\mathbb{R}^{2}\right)}+\left\|\partial^{\alpha}(\eta \nabla \varphi)\right\|_{L^{q}\left(\mathbb{R}^{2}\right)}\right) .
$$

For $\alpha=(0,0)$, inequality (3.16) is a direct consequence of (3.19) invoking (3.3). For $\alpha \neq(0,0)$, the derivative $\partial^{\alpha}(\eta \nabla \varphi)$ may be written as

$$
\partial^{\alpha}(\eta \nabla \varphi)=\sum_{0 \leq \beta \leq \alpha}\left(\begin{array}{l}
\alpha \\
\beta
\end{array}\right) \partial^{\beta} \eta \partial^{\alpha-\beta}(\nabla \varphi),
$$


by Leibniz formula, so that

$$
\begin{aligned}
\left\|\partial^{\alpha}(\eta \nabla \varphi)\right\|_{L^{q}\left(\mathbb{R}^{2}\right)} \leq & K(q, \alpha)\left(\left\|\partial^{\alpha} \eta\right\|_{L^{q}\left(\mathbb{R}^{2}\right)}\|\nabla \varphi\|_{L^{\infty}\left(\mathbb{R}^{2}\right)}\right. \\
& \left.+\sum_{0 \leq \beta<\alpha}\left\|\partial^{\beta} \eta\right\|_{L^{\infty}\left(\mathbb{R}^{2}\right)}\left\|\partial^{\alpha-\beta}(\nabla \varphi)\right\|_{L^{q}\left(\mathbb{R}^{2}\right)}\right) .
\end{aligned}
$$

Estimate (3.17) follows from (3.19) using again uniform bound (3.3).

3.2. Properties of $u_{\mathfrak{p}}$. We now restrict ourselves to the solutions $u_{\mathfrak{p}}$ provided by Theorem 1 . We begin with the

Proof of Lemma 1. In view of (3), we have

$$
\Sigma_{\mathfrak{p}} \equiv \Sigma\left(u_{\mathfrak{p}}\right) \geq \frac{48 \sqrt{2}}{\mathcal{S}_{K P}^{2}} \mathfrak{p}^{3}-K_{0} \mathfrak{p}^{4},
$$

for any $\mathfrak{p}$ sufficiently small, whereas, by $(3.13)$,

$$
\Sigma_{\mathfrak{p}} \leq \mathfrak{p} \frac{\varepsilon_{\mathfrak{p}}^{2}}{\sqrt{2}}
$$

so that, combining both the inequalities, we obtain

$$
\varepsilon_{\mathfrak{p}} \geq \frac{9}{\mathcal{S}_{K P}} \mathfrak{p}
$$

On the other hand, in view of Proposition 1, we have

$$
\varepsilon_{\mathfrak{p}} \leq K E_{\mathfrak{p}},
$$

where we have set $E_{\mathfrak{p}} \equiv E\left(u_{\mathfrak{p}}\right)$. Since $E_{\mathfrak{p}} \leq \sqrt{2} \mathfrak{p}$, we conclude that (10) holds. Moreover, we also have

$$
K_{5} E_{\mathfrak{p}} \leq \varepsilon_{\mathfrak{p}} \leq K_{6} E_{\mathfrak{p}},
$$

for any $\mathfrak{p}$ sufficiently small, and some positive constants $K_{5}$ and $K_{6}$, not depending on $\mathfrak{p}$.

Finally, since $\Sigma_{\mathfrak{p}}>0$ by (3), we deduce from Lemma 1 that (3.12), (3.13) and (3.14) may be recast as

$$
\int_{\mathbb{R}^{2}}\left(\left|\nabla \varrho_{\mathfrak{p}}\right|^{2}+\left(\partial_{2} u_{\mathfrak{p}}\right)^{2}\right)+\left.\left|\int_{\mathbb{R}^{2}} \eta_{\mathfrak{p}}\right| \nabla \varphi_{\mathfrak{p}}\right|^{2} \mid \leq K \mathfrak{p}^{3},
$$

where we denote $u_{\mathfrak{p}}=\varrho_{\mathfrak{p}} \exp i \varphi_{\mathfrak{p}}$. Since $\left(\partial_{2} u_{\mathfrak{p}}\right)^{2}=\varrho_{\mathfrak{p}}^{2}\left(\partial_{2} \varphi_{\mathfrak{p}}\right)^{2}+\left(\partial_{2} \varrho_{\mathfrak{p}}\right)^{2}$ and $\left|\eta_{\mathfrak{p}}\right| \leq$ $3 \varrho_{\mathfrak{p}}^{2}$, we deduce that

$$
\int_{\mathbb{R}^{2}}\left|\eta_{\mathfrak{p}}\right|\left(\partial_{2} \varphi_{\mathfrak{p}}\right)^{2} \leq 3 \int_{\mathbb{R}^{2}} \varrho_{\mathfrak{p}}^{2}\left(\partial_{2} \varphi_{\mathfrak{p}}\right)^{2} \leq K \mathfrak{p}^{3}
$$

so that

$$
\left|\int_{\mathbb{R}^{2}} \eta_{\mathfrak{p}}\left(\partial_{1} \varphi_{\mathfrak{p}}\right)^{2}\right| \leq K \mathfrak{p}^{3}
$$




\section{4. (TWc) in the slow space variables}

4.1. Expansion of the energy functionals. In this subsection, we consider a finite energy map $v$ on $\mathbb{R}^{2}$, satisfying (3.6), and a small given parameter $\varepsilon>0$. In view of assumption (3.6), we may lift $v$ as $v=\varrho \exp i \varphi$. Following the expansion given in the physical literature, we introduce anisotropic slow space variables $\tilde{x}_{1}=$ $\varepsilon x_{1}$, and $\tilde{x}_{2}=\frac{\varepsilon^{2}}{\sqrt{2}} x_{2}$. We then consider the rescaled functions $N=N_{v, \varepsilon}$ and $\Theta=\Theta_{v, \varepsilon}$ defined as follows

$$
N(x)=\frac{6}{\varepsilon^{2}} \eta\left(\frac{x_{1}}{\varepsilon}, \frac{\sqrt{2} x_{2}}{\varepsilon^{2}}\right), \text { and } \Theta(x)=\frac{6 \sqrt{2}}{\varepsilon} \varphi\left(\frac{x_{1}}{\varepsilon}, \frac{\sqrt{2} x_{2}}{\varepsilon^{2}}\right) .
$$

We next express the functionals $p$ and $E$ in terms of the functions $N, \Theta$ and $\varepsilon$. In the course of the analysis, we will also compute several other integral quantities in the rescaled variables. For instance,

$$
\begin{gathered}
\int_{\mathbb{R}^{2}} N^{2}=\frac{18 \sqrt{2}}{\varepsilon} \int_{\mathbb{R}^{2}} \eta^{2}, \int_{\mathbb{R}^{2}}\left(\partial_{1} N\right)^{2}=\frac{18 \sqrt{2}}{\varepsilon^{3}} \int_{\mathbb{R}^{2}}\left(\partial_{1} \eta\right)^{2} \\
\int_{\mathbb{R}^{2}}\left(\partial_{2} N\right)^{2}=\frac{36 \sqrt{2}}{\varepsilon^{5}} \int_{\mathbb{R}^{2}}\left(\partial_{2} \eta\right)^{2}
\end{gathered}
$$

whereas

$$
\int_{\mathbb{R}^{2}}\left(\partial_{1} \Theta\right)^{2}=\frac{36 \sqrt{2}}{\varepsilon} \int_{\mathbb{R}^{2}}\left(\partial_{1} \varphi\right)^{2}, \text { and } \int_{\mathbb{R}^{2}}\left(\partial_{2} \Theta\right)^{2}=\frac{72 \sqrt{2}}{\varepsilon^{3}} \int_{\mathbb{R}^{2}}\left(\partial_{2} \varphi\right)^{2} .
$$

A rather tedious computation along the same lines allows to derive the following expansions.

LEMMA 4.1. Let $v$ be a smooth map on $\mathbb{R}^{2}$ satisfying (3.6), and let $N$ and $\Theta$ be the corresponding functions defined by (4.1). The momentum $p(v)$ can be expressed in terms of the new functions as

$$
p(v)=\frac{\varepsilon}{72} \int_{\mathbb{R}^{2}} N \partial_{1} \Theta
$$

while the energy $E(v)$ has the expansion

$$
E(v)=\sqrt{2} \frac{\varepsilon}{144}\left(E_{0}(N, \Theta)+\varepsilon^{2} E_{2}(N, \Theta)+\varepsilon^{4} E_{4}(N, \Theta)\right),
$$

where the functions $E_{0}, E_{2}$ and $E_{4}$ are given by

$$
E_{2}(N, \Theta)=\int_{\mathbb{R}^{2}}\left(\frac{1}{2}\left(\partial_{1} N\right)^{2}+\frac{1}{2}\left(\partial_{2} \Theta\right)^{2}-\frac{1}{6} N\left(\partial_{1} \Theta\right)^{2}\right)
$$

and

$$
E_{4}(N, \Theta)=\int_{\mathbb{R}^{2}}\left(\frac{\left(\partial_{2} N\right)^{2}}{4-\frac{2 \varepsilon^{2}}{3} N}+\frac{N\left(\partial_{1} N\right)^{2}}{12-2 \varepsilon^{2} N}-\frac{1}{12} N\left(\partial_{2} \Theta\right)^{2}\right) .
$$

REmark 4.1. Recall that the map $u_{\mathfrak{p}}$ found in Theorem 1 minimizes the Ginzburg-Landau energy keeping the momentum $p$ fixed, equal to $\mathfrak{p}$. If one takes instead only the first term of the energy in expansion (4.3), i.e. if one minimizes 
$E_{0}$ keeping the momentum $p$ equal to $\mathfrak{p}$, then $\tilde{u}_{\mathfrak{p}}$ will be a minimizer for the new problem if and only if

$$
\tilde{N}_{\mathfrak{p}}=\partial_{1} \tilde{\Theta}_{\mathfrak{p}}, \text { and } \int_{\mathbb{R}^{2}} \tilde{N}_{\mathfrak{p}}^{2}=\frac{72 \mathfrak{p}}{\varepsilon}
$$

Notice in particular that $\tilde{\Theta}_{\mathfrak{p}}=\partial_{1}^{-1} \tilde{N}_{\mathfrak{p}}$, so that $\partial_{1}^{-1}\left(\partial_{2} \tilde{N}_{\mathfrak{p}}\right)=\partial_{2} \tilde{\Theta}_{\mathfrak{p}}$. If we insert these relations into the definition of $E_{2}\left(\tilde{N}_{\mathfrak{p}}, \tilde{\Theta}_{\mathfrak{p}}\right)$, one obtains

$$
E_{2}\left(\tilde{N}_{\mathfrak{p}}, \tilde{\Theta}_{\mathfrak{p}}\right)=\int_{\mathbb{R}^{2}}\left(\frac{1}{2}\left(\partial_{1} \tilde{N}_{\mathfrak{p}}\right)^{2}+\frac{1}{2}\left(\partial_{1}^{-1}\left(\partial_{2} \tilde{N}_{\mathfrak{p}}\right)\right)^{2}-\frac{1}{6} \tilde{N}_{\mathfrak{p}}^{3}\right)=E_{K P}\left(\tilde{N}_{\mathfrak{p}}\right)
$$

This identity gives a first heuristic relation between the (GP) functional and the (KP I) functional, as well as between the solutions $u_{\mathfrak{p}}$ and the ground states for (KP I).

Specifying the above change of variables to the case $v=u_{\mathfrak{p}}$ and $\varepsilon=\varepsilon_{\mathfrak{p}}$, setting $N_{\mathfrak{p}}=N_{u_{\mathfrak{p}}, \varepsilon_{\mathfrak{p}}}$ and $\Theta_{\mathfrak{p}}=\Theta_{u_{\mathfrak{p}}, \varepsilon_{\mathfrak{p}}}$, we obtain bounds for the integral quantities appearing in Lemma 4.1. In view of (1) and (3.20), we have

$$
\int_{\mathbb{R}^{2}}\left(N_{\mathfrak{p}}\right)^{2}=\frac{18 \sqrt{2}}{\varepsilon_{\mathfrak{p}}} \int_{\mathbb{R}^{2}} \eta_{\mathfrak{p}}^{2} \leq \frac{72 \sqrt{2} E\left(u_{\mathfrak{p}}\right)}{\varepsilon_{\mathfrak{p}}} \leq K
$$

where $K$ is some universal constant, whereas by (3.6) and (3.7),

$$
\int_{\mathbb{R}^{2}}\left(\partial_{1} \Theta_{\mathfrak{p}}\right)^{2}=\frac{36 \sqrt{2}}{\varepsilon_{\mathfrak{p}}} \int_{\mathbb{R}^{2}}\left(\partial_{1} \varphi_{\mathfrak{p}}\right)^{2} \leq \frac{144 \sqrt{2}}{\varepsilon_{\mathfrak{p}}} \int_{\mathbb{R}^{2}} \varrho_{\mathfrak{p}}^{2}\left(\partial_{1} \varphi_{\mathfrak{p}}\right)^{2} \leq \frac{288 \sqrt{2} E\left(u_{\mathfrak{p}}\right)}{\varepsilon_{\mathfrak{p}}},
$$

so that

$$
\int_{\mathbb{R}^{2}}\left(\left(N_{\mathfrak{p}}\right)^{2}+\left(\partial_{1} \Theta_{\mathfrak{p}}\right)^{2}\right) \leq K
$$

Similarly, it follows from (3.21) and (3.22) that

$$
\int_{\mathbb{R}^{2}}\left(\left(\partial_{1} N_{\mathfrak{p}}\right)^{2}+\left(\partial_{2} \Theta_{\mathfrak{p}}\right)^{2}\right)+\left|\int_{\mathbb{R}^{2}} N_{\mathfrak{p}}\left(\partial_{1} \Theta_{\mathfrak{p}}\right)^{2}\right| \leq K
$$

For various other quantities, we only have at this stage rather crude estimates. For instance, concerning the uniform norm of $N_{\mathfrak{p}}$, the bound provided by (3.3) yields

$$
\left\|N_{\mathfrak{p}}\right\|_{L \infty\left(\mathbb{R}^{2}\right)} \leq \frac{K}{\varepsilon_{\mathfrak{p}}^{2}}
$$

We also only have for the transverse derivatives

$$
\int_{\mathbb{R}^{2}}\left(\partial_{2} N_{\mathfrak{p}}\right)^{2}+\int_{\mathbb{R}^{2}}\left|N_{\mathfrak{p}}\left(\partial_{2} \Theta_{\mathfrak{p}}\right)^{2}\right| \leq \frac{K}{\varepsilon_{\mathfrak{p}}^{2}}
$$

It follows from (4.8) that

$$
\left|E_{2}\left(N_{\mathfrak{p}}, \Theta_{\mathfrak{p}}\right)\right| \leq K
$$

whereas for $E_{4}$, we only obtain combining estimates (4.8), (4.9) and (4.10),

$$
\left|E_{4}\left(N_{\mathfrak{p}}, \Theta_{\mathfrak{p}}\right)\right| \leq \frac{K}{\varepsilon_{\mathfrak{p}}^{2}} .
$$

Hence, going back to the expansion of the energy, we deduce

$$
\left|E\left(u_{\mathfrak{p}}\right)-\sqrt{2} \frac{\varepsilon_{\mathfrak{p}}}{144} E_{0}\left(N_{\mathfrak{p}}, \Theta_{\mathfrak{p}}\right)\right| \leq K \varepsilon_{\mathfrak{p}}^{3}
$$

This leads to 
LEMma 4.2. There exists some positive constant $K$, not depending on $\mathfrak{p}$, such that

$$
\int_{\mathbb{R}^{2}}\left(N_{\mathfrak{p}}-\partial_{1} \Theta_{\mathfrak{p}}\right)^{2} \leq K \varepsilon_{\mathfrak{p}}^{2}
$$

for any $\mathfrak{p}$ sufficiently small.

Proof. Using (4.2), (4.4) and (4.11), we are led to

$$
\int_{\mathbb{R}^{2}}\left(N_{\mathfrak{p}}-\partial_{1} \Theta_{\mathfrak{p}}\right)^{2}=E_{0}\left(N_{\mathfrak{p}}, \Theta_{\mathfrak{p}}\right)-2 \int_{\mathbb{R}^{2}} N_{\mathfrak{p}} \partial_{1} \Theta_{\mathfrak{p}} \leq \frac{144 E\left(u_{\mathfrak{p}}\right)}{\sqrt{2} \varepsilon_{\mathfrak{p}}}-\frac{144 \mathfrak{p}}{\varepsilon_{\mathfrak{p}}}+K \varepsilon_{\mathfrak{p}}^{2} .
$$

Since $E\left(u_{\mathfrak{p}}\right) \leq \sqrt{2} \mathfrak{p}$, the conclusion follows.

Estimate (4.7) provides a first step to compactness. In particular, there exists some map $N_{0} \in L^{2}\left(\mathbb{R}^{2}\right)$ such that, up to a subsequence,

$$
N_{\mathfrak{p}} \rightarrow N_{0} \text { in } L^{2}\left(\mathbb{R}^{2}\right), \text { as } \mathfrak{p} \rightarrow 0 .
$$

As a consequence of Lemma 4.2, we also have

$$
\partial_{1} \Theta_{\mathfrak{p}} \rightarrow N_{0} \text { in } L^{2}\left(\mathbb{R}^{2}\right), \text { as } \mathfrak{p} \rightarrow 0
$$

To improve this convergence and characterize the limit function $N_{0}$, we turn to the equations for $N_{\mathfrak{p}}$ and $\Theta_{\mathfrak{p}}$.

4.2. Expansion of the equations. We now consider a finite energy solution $v$ to (TWc) satisfying (3.6), so that $v$ may be written as $v=\varrho \exp i \varphi$, and the functions $\varrho$ and $\varphi$ satisfy the system of equations (3.8)-(3.9). At first order, each of the equations (3.8) and (3.9) express the fact that

$$
N \sim \partial_{1} \Theta, \text { as } \varepsilon \rightarrow 0 .
$$

Indeed, we first have

LEMMA 4.3. Assume $\varrho$ and $\varphi$ satisfy (3.9), and let $N$ and $\Theta$ be the corresponding functions defined by (4.1). Then, $N$ and $\Theta$ satisfy

$$
N-\partial_{1} \Theta=\varepsilon^{2}\left(\mathcal{L}_{\varepsilon, 1}(N, \Theta)+\mathcal{R}_{\varepsilon, 1}(N, \Theta)\right),
$$

where the remainder terms $\mathcal{L}_{\varepsilon, 1}(N, \Theta)$ and $\mathcal{R}_{\varepsilon, 1}(N, \Theta)$ are given by

$$
\mathcal{L}_{\varepsilon, 1}(N, \Theta)=\frac{1}{\varepsilon^{2}}\left(\sqrt{1-\frac{\varepsilon^{2}}{2}}-1\right) \partial_{1} \Theta+\frac{1}{2} \partial_{1}^{2} N+\frac{\varepsilon^{2}}{4} \partial_{2}^{2} N,
$$

and

$$
\begin{aligned}
\mathcal{R}_{\varepsilon, 1}(N, \Theta) & =\frac{1}{12}\left(2 N^{2}-2 \sqrt{1-\frac{\varepsilon^{2}}{2}} N \partial_{1} \Theta+\left(\partial_{1} \Theta\right)^{2}\right) \\
& +\frac{\varepsilon^{2}}{72}\left(3 \frac{\left(\partial_{1} N\right)^{2}}{1-\frac{\varepsilon^{2}}{6} N}-N\left(\partial_{1} \Theta\right)^{2}+3\left(\partial_{2} \Theta\right)^{2}\right) \\
& +\frac{\varepsilon^{4}}{144}\left(3 \frac{\left(\partial_{2} N\right)^{2}}{1-\frac{\varepsilon^{2}}{6} N}-N\left(\partial_{2} \Theta\right)^{2}\right) .
\end{aligned}
$$

We similarly have 
LEMma 4.4. Assume $\varrho$ and $\varphi$ satisfy (3.8), and let $N$ and $\Theta$ be the corresponding functions defined by (4.1). Then, $N$ and $\Theta$ satisfy

$$
\partial_{1} N-\partial_{1}^{2} \Theta=\varepsilon^{2}\left(\mathcal{L}_{\varepsilon, 2}(N, \Theta)+\mathcal{R}_{\varepsilon, 2}(N, \Theta)\right),
$$

where the remainder terms $\mathcal{L}_{\varepsilon, 2}(N, \Theta)$ and $\mathcal{R}_{\varepsilon, 2}(N, \Theta)$ are given by

$$
\mathcal{L}_{\varepsilon, 2}(N, \Theta)=\frac{1}{\varepsilon^{2}}\left(1-\sqrt{1-\frac{\varepsilon^{2}}{2}}\right) \partial_{1} N+\frac{1}{2} \partial_{2}^{2} \Theta
$$

and

$$
\mathcal{R}_{\varepsilon, 2}(N, \Theta)=-\frac{1}{6} \partial_{1}\left[N \partial_{1} \Theta\right]-\frac{\varepsilon^{2}}{12} \partial_{2}\left[N \partial_{2} \Theta\right]
$$

As mentioned above, equations (4.13) and (4.14) twice express the fact that the functions $N$ and $\partial_{1} \Theta$ are equal at the limit $\varepsilon \rightarrow 0$. In order to identify their common limit, we expand some combination of (4.13) and (4.14) to deduce

Proposition 4.1. Let $v$ be a finite energy solution to (TWc) on $\mathbb{R}^{2}$ satisfying (3.6), and let $N$ and $\Theta$ be the corresponding functions defined by (4.1). Then, $N$ and $\Theta$ satisfy

$$
\mathcal{L}(N)=-\partial_{1}^{2}\left[\frac{1}{3} N^{2}+\frac{1}{6}\left(\partial_{1} \Theta\right)^{2}\right]+\varepsilon^{2}\left(\mathcal{L}_{\varepsilon}(N)+\mathcal{R}_{\varepsilon}(N, \Theta)\right),
$$

where $\mathcal{L}$ is the linear operator given by

$$
\mathcal{L}(N)=\partial_{1}^{4} N-\Delta N,
$$

and the remainder terms $\mathcal{L}_{\varepsilon}(N)$ and $\mathcal{R}_{\varepsilon}(N, \Theta)$ are given by

$$
\mathcal{L}_{\varepsilon}(N)=-\partial_{1}^{2} \partial_{2}^{2} N-\frac{\varepsilon^{2}}{4} \partial_{2}^{4} N
$$

and

$$
\begin{aligned}
\mathcal{R}_{\varepsilon}(N, \Theta)= & \frac{1}{72}\left(2 \partial_{1}^{2}\left[N\left(\partial_{1} \Theta\right)^{2}\right]-6 \partial_{1}^{2}\left[\frac{\left(\partial_{1} N\right)^{2}}{1-\frac{\varepsilon^{2}}{6} N}\right]\right. \\
& -24 \partial_{2}^{2}\left(N^{2}\right)-6 \sqrt{1-\frac{\varepsilon^{2}}{2}} \partial_{1} \partial_{2}\left[N \partial_{2} \Theta\right] \\
& \left.+12 \sqrt{1-\frac{\varepsilon^{2}}{2}} \partial_{2}^{2}\left[N \partial_{1} \Theta\right]-3 \partial_{1}^{2}\left[\left(\partial_{2} \Theta\right)^{2}\right]-6 \partial_{2}^{2}\left[\left(\partial_{1} \Theta\right)^{2}\right]\right) \\
& +\frac{\varepsilon^{2}}{144}\left(-3 \partial_{1}^{2}\left[\frac{\left(\partial_{2} N\right)^{2}}{1-\frac{\varepsilon^{2}}{6} N}\right]+\partial_{1}^{2}\left[N\left(\partial_{2} \Theta\right)^{2}\right]\right. \\
& -6 \partial_{2}^{2}\left[\frac{\left(\partial_{1} N\right)^{2}}{1-\frac{\varepsilon^{2}}{6} N}\right]+2 \partial_{2}^{2}\left[N\left(\partial_{1} \Theta\right)^{2}\right] \\
& \left.-3 \partial_{2}^{2}\left[\left(\partial_{2} \Theta\right)^{2}\right]\right)+\frac{\varepsilon^{4}}{288}\left(-3 \partial_{2}^{2}\left[\frac{\left(\partial_{2} N\right)^{2}}{1-\frac{\varepsilon^{2}}{6} N}\right]+\partial_{2}^{2}\left[N\left(\partial_{2} \Theta\right)^{2}\right]\right) .
\end{aligned}
$$

Proof. Equation (4.15) is derived applying the differential operator $-\partial_{1}^{2}-$ $\frac{\varepsilon^{2}}{2} \partial_{2}^{2}$ to $(4.13)$, the operator $\sqrt{1-\frac{\varepsilon^{2}}{2}} \partial_{1}$ to $(4.14)$, and adding the corresponding relations. 
Notice that we have at this stage,

$$
\partial_{1}^{4} N-\Delta N+\frac{1}{2} \partial_{1}^{2} N^{2}=\frac{1}{6} \partial_{1}^{2}\left(N^{2}-\left(\partial_{1} \Theta\right)^{2}\right)+\varepsilon^{2}\left(\mathcal{L}_{\varepsilon}(N, \Theta)+\mathcal{R}_{\varepsilon}(N, \Theta)\right)
$$

where we recognize equation (2.1) for $N$ in the left-hand side. Specifying this relation to the solutions $N_{\mathfrak{p}}$ and $\Theta_{\mathfrak{p}}$, it remains to prove that the weak limit $N_{0}$ of the sequence $\left(N_{\mathfrak{p}}\right)_{\mathfrak{p}>0}$ is a solution to $(\mathrm{SW})$, and to show some strong convergence. This requires to establish that the nonlinear remainder term $\mathcal{R}_{\varepsilon}$ is small in some suitable sense. Indeed, the first term on the right-hand side will tend to 0 in view of Lemma 4.2, whereas the linear term $\mathcal{L}_{\varepsilon}(N)$ presents no difficulty.

The remainder term $\mathcal{R}_{\varepsilon}$ is a sum of several second order derivatives. We order them according to the type of second order derivatives, writing

$$
\mathcal{R}_{\varepsilon}(N, \Theta)=\sum_{i+j=2} \partial_{1}^{i} \partial_{2}^{j} \mathcal{R}_{\varepsilon}^{i, j}
$$

where

$$
\begin{gathered}
\mathcal{R}_{\varepsilon}^{2,0}=\frac{1}{36} N\left(\partial_{1} \Theta\right)^{2}-\frac{\left(\partial_{1} N\right)^{2}}{12\left(1-\frac{\varepsilon^{2}}{6} N\right)}-\frac{1}{24}\left(\partial_{2} \Theta\right)^{2} \\
-\varepsilon^{2} \frac{\left(\partial_{2} N\right)^{2}}{48\left(1-\frac{\varepsilon^{2}}{6} N\right)}+\frac{\varepsilon^{2}}{144} N\left(\partial_{2} \Theta\right)^{2} \\
\mathcal{R}_{\varepsilon}^{0,2}=-\frac{N^{2}}{3}+\frac{1}{6} \sqrt{1-\frac{\varepsilon^{2}}{2}} N \partial_{1} \Theta-\frac{\left(\partial_{1} \Theta\right)^{2}}{12}-\varepsilon^{2} \frac{\left(\partial_{1} N\right)^{2}}{24\left(1-\frac{\varepsilon^{2}}{6} N\right)} \\
+\frac{\varepsilon^{2}}{72} N\left(\partial_{1} \Theta\right)^{2}-\frac{\varepsilon^{2}}{48}\left(\partial_{2} \Theta\right)^{2}-\varepsilon^{4} \frac{\left(\partial_{2} N\right)^{2}}{96\left(1-\frac{\varepsilon^{2}}{6} N\right)}+\frac{\varepsilon^{4}}{288} N\left(\partial_{2} \Theta\right)^{2},
\end{gathered}
$$

and

$$
\mathcal{R}_{\varepsilon}^{1,1}=-\frac{1}{12} \sqrt{1-\frac{\varepsilon^{2}}{2}} N \partial_{2} \Theta .
$$

In several places, it will be convenient to write

$$
\mathcal{R}_{\varepsilon}^{i, j}=R_{\varepsilon}^{i, j}+\varepsilon^{2} \nu_{\varepsilon}^{i, j},
$$

where $\nu_{\varepsilon}^{1,1}=0$,

$$
\nu_{\varepsilon}^{2,0}=-\frac{\left(\partial_{2} N\right)^{2}}{48\left(1-\frac{\varepsilon^{2}}{6} N\right)}+\frac{1}{144} N\left(\partial_{2} \Theta\right)^{2}
$$

and

$\nu_{\varepsilon}^{0,2}=-\frac{\left(\partial_{1} N\right)^{2}}{24\left(1-\frac{\varepsilon^{2}}{6} N\right)}+\frac{1}{72} N\left(\partial_{1} \Theta\right)^{2}-\frac{1}{48}\left(\partial_{2} \Theta\right)^{2}-\varepsilon^{2} \frac{\left(\partial_{2} N\right)^{2}}{96\left(1-\frac{\varepsilon^{2}}{6} N\right)}+\frac{\varepsilon^{2}}{288} N\left(\partial_{2} \Theta\right)^{2}$.

Notice in particular that

$$
\left|R_{\varepsilon}^{2,0}\right| \leq K\left(\left(\partial_{1} N\right)^{2}+\left(\partial_{2} \Theta\right)^{2}+\left|N\left(\partial_{1} \Theta\right)^{2}\right|\right),
$$

whereas

$$
\left|R_{\varepsilon}^{1,1}\right| \leq K|N|\left|\partial_{2} \Theta\right|, \text { and }\left|R_{\varepsilon}^{0,2}\right| \leq K\left(N^{2}+\left(\partial_{1} \Theta\right)^{2}\right)
$$


Similarly, we also have

$$
\begin{aligned}
& \left|\nu_{\varepsilon}^{2,0}\right| \leq K\left(\left(\partial_{2} N\right)^{2}+\left|N\left(\partial_{2} \Theta\right)^{2}\right|\right) \\
& \left|\nu_{\varepsilon}^{0,2}\right| \leq K\left(\left(\partial_{1} N\right)^{2}+\left|N\left(\partial_{1} \Theta\right)^{2}\right|+\left(\partial_{2} \Theta\right)^{2}+\varepsilon^{2}\left(\left(\partial_{2} N\right)^{2}+\left|N\left(\partial_{2} \Theta\right)^{2}\right|\right)\right) .
\end{aligned}
$$

Specifying the previous quantities for $N_{\mathfrak{p}}$ and $\Theta_{\mathfrak{p}}$, we obtain some initial bounds on the nonlinear remainder terms, which will prove essential to compute the estimates of Proposition 3.

LEMMA 4.5. There exists some positive constant $K$, not depending on $\mathfrak{p}$, such that

$$
\int_{\mathbb{R}^{2}}\left(\left|\mathcal{R}_{\varepsilon_{\mathfrak{p}}}^{1,1}\right|+\left|R_{\varepsilon_{\mathfrak{p}}}^{0,2}\right|\right) \leq K
$$

and

$$
\int_{\mathbb{R}^{2}}\left(\left|R_{\varepsilon_{\mathfrak{p}}}^{2,0}\right|+\left|\nu_{\varepsilon_{\mathfrak{p}}}^{2,0}\right|+\left|\nu_{\varepsilon_{\mathfrak{p}}}^{0,2}\right|\right) \leq \frac{K}{\varepsilon_{\mathfrak{p}}^{2}},
$$

for any $\mathfrak{p}$ sufficiently small.

Proof. Bounds (4.22) and (4.23) are consequences of bounds (4.7), (4.8) and (4.10), and inequalities (4.19), (4.20) and (4.21). Concerning the term $\int_{\mathbb{R}^{2}} N_{\mathfrak{p}}\left(\partial_{1} \Theta_{\mathfrak{p}}\right)^{2}$ in (4.23), we have to invoke the crude bound (4.9), which yields

$$
\int_{\mathbb{R}^{2}}\left|N_{\mathfrak{p}}\left(\partial_{1} \Theta_{\mathfrak{p}}\right)^{2}\right| \leq \frac{K}{\varepsilon_{\mathfrak{p}}^{2}} \int_{\mathbb{R}^{2}}\left(\partial_{1} \Theta_{\mathfrak{p}}\right)^{2} \leq \frac{K}{\varepsilon_{\mathfrak{p}}^{2}} .
$$

4.3. Estimates for the phase $\Theta_{\mathfrak{p}}$. In the previous discussion, we did not consider the function $\Theta$. In particular, we did not compute any rescaled equation for this function. Applying the partial differential operator $\mathcal{L}-\varepsilon^{2} \mathcal{L}_{\varepsilon}$ to $(4.14)$ and introducing equation (4.15) in the resulting equation in order to eliminate the function $N$ in the linear part, we compute

$$
\mathcal{L}\left(\partial_{1}^{2} \Theta\right)=-\partial_{1}^{3}\left(\frac{1}{3} N^{2}+\frac{1}{6}\left(\partial_{1} \Theta\right)^{2}\right)+\varepsilon^{2}\left(\mathcal{L}_{\varepsilon, 3}(\Theta)+\mathcal{R}_{\varepsilon, 3}(N, \Theta)\right)
$$

where the remainder terms $\mathcal{L}_{\varepsilon, 3}(\Theta)$ and $\mathcal{R}_{\varepsilon, 3}(N, \Theta)$ are given by

$$
\mathcal{L}_{\varepsilon, 3}(\Theta)=\mathcal{L}_{\varepsilon}\left(\partial_{1}^{2} \Theta\right)-\frac{1}{2} \mathcal{L}\left(\partial_{2}^{2} \Theta\right)+\frac{\varepsilon^{2}}{2} \mathcal{L}_{\varepsilon}\left(\partial_{2}^{2} \Theta\right)
$$

and

$$
\begin{aligned}
\mathcal{R}_{\varepsilon, 3}(N, \Theta) & =\frac{1}{\varepsilon^{2}}\left(1-\sqrt{1-\frac{\varepsilon^{2}}{2}}\right) \partial_{1}^{3}\left(\frac{1}{3} N^{2}+\frac{1}{6}\left(\partial_{1} \Theta\right)^{2}\right)+\sqrt{1-\frac{\varepsilon^{2}}{2}} \partial_{1} \mathcal{R}_{\varepsilon}(N, \Theta) \\
& -\mathcal{L}\left(\mathcal{R}_{\varepsilon, 2}(N, \Theta)\right)+\varepsilon^{2} \mathcal{L}_{\varepsilon}\left(\mathcal{R}_{\varepsilon, 2}(N, \Theta)\right) .
\end{aligned}
$$

At least formally, this may be written as

$\partial_{1}^{4}\left(\partial_{1} \Theta\right)-\Delta\left(\partial_{1} \Theta\right)+\frac{1}{2} \partial_{1}^{2}\left(\partial_{1} \Theta\right)^{2}=\frac{1}{3} \partial_{1}^{2}\left(\left(\partial_{1} \Theta\right)^{2}-N^{2}\right)+\varepsilon^{2} \partial_{1}^{-1}\left(\mathcal{L}_{\varepsilon, 3}(\Theta)+\mathcal{R}_{\varepsilon, 3}(N, \Theta)\right)$.

We recognize once more equation (2.1) for $\partial_{1} \Theta$ in the left-hand side. However, the analysis of equation (4.24) is substantially more difficult than the study of (4.15), due to the intricacy of the remainder terms and the necessity to apply the operator $\partial_{1}^{-1}$ to (4.24) to recover (2.1). Hence, our argument to deal with the phase $\Theta$ does 
not rely on (4.24). Instead, we invoke the estimates of Lemma 3.3, whose rescaled versions give bounds on $\Theta$ in function of those on $N$.

Lemma 4.6. Let $1<q<+\infty$. There exists some positive constant $K(q)$, not depending on $\mathfrak{p}$, such that

$$
\left\|\partial_{1} \Theta_{\mathfrak{p}}\right\|_{L^{q}\left(\mathbb{R}^{2}\right)}+\varepsilon_{\mathfrak{p}}\left\|\partial_{2} \Theta_{\mathfrak{p}}\right\|_{L^{q}\left(\mathbb{R}^{2}\right)} \leq K(q)\left\|N_{\mathfrak{p}}\right\|_{L^{q}\left(\mathbb{R}^{2}\right)},
$$

for any $\mathfrak{p}$ sufficiently small. Similarly, given any $\alpha \in \mathbb{N}^{2}$, and denoting

$$
\Xi_{\mathfrak{p}}(q, \alpha) \equiv\left\|\partial^{\alpha} \partial_{1} \Theta_{\mathfrak{p}}\right\|_{L^{q}\left(\mathbb{R}^{2}\right)}+\varepsilon_{\mathfrak{p}}\left\|\partial^{\alpha} \partial_{2} \Theta_{\mathfrak{p}}\right\|_{L^{q}\left(\mathbb{R}^{2}\right)}
$$

there exists some positive constant $K(q, \alpha)$, not depending on $\mathfrak{p}$, such that

$$
\Xi_{\mathfrak{p}}(q, \alpha) \leq K(q, \alpha)\left(\left\|\partial^{\alpha} N_{\mathfrak{p}}\right\|_{L^{q}\left(\mathbb{R}^{2}\right)}+\varepsilon_{\mathfrak{p}}^{2} \sum_{0 \leq \beta<\alpha}\left\|\partial^{\beta} N_{\mathfrak{p}}\right\|_{L^{\infty}\left(\mathbb{R}^{2}\right)} \Xi_{\mathfrak{p}}(q, \alpha-\beta)\right)
$$

for any $\mathfrak{p}$ sufficiently small.

Proof. Inequalities (4.25) and (4.26) are rescaled versions of (3.16) and (3.17). In view of scalings (4.1), given any $1<q \leq+\infty$, the $L^{q}$-norm of the function $\partial^{\alpha} N$ is related to the $L^{q}$-norm of $\partial^{\alpha} \eta$ by

$$
\left\|\partial^{\alpha} N\right\|_{L^{q}\left(\mathbb{R}^{2}\right)}=\frac{K(q, \alpha)}{\varepsilon^{2+\alpha_{1}+2 \alpha_{2}-\frac{3}{q}}}\left\|\partial^{\alpha} \eta\right\|_{L^{q}\left(\mathbb{R}^{2}\right)},
$$

where $K(q, \alpha)$ denotes some positive constant, not depending on $\varepsilon$. Similarly, we compute for the functions $\partial^{\alpha} \partial_{1} \Theta$ and $\partial^{\alpha} \partial_{2} \Theta$,

$$
\begin{aligned}
& \left\|\partial^{\alpha} \partial_{1} \Theta\right\|_{L^{q}\left(\mathbb{R}^{2}\right)}=\frac{K(q, \alpha)}{\varepsilon^{2+\alpha_{1}+2 \alpha_{2}-\frac{3}{q}}}\left\|\partial^{\alpha} \partial_{1} \varphi\right\|_{L^{q}\left(\mathbb{R}^{2}\right)}, \\
& \text { and }\left\|\partial^{\alpha} \partial_{2} \Theta\right\|_{L^{q}\left(\mathbb{R}^{2}\right)}=\frac{K(q, \alpha)}{\varepsilon^{3+\alpha_{1}+2 \alpha_{2}-\frac{3}{q}}}\left\|\partial^{\alpha} \varphi\right\|_{L^{q}\left(\mathbb{R}^{2}\right)} .
\end{aligned}
$$

Inequalities (4.25) and (4.26) then follow from rescaling (3.16) and (3.17), specifying identities (4.27) and (4.28) for the functions $N_{\mathfrak{p}}$ and $\Theta_{\mathfrak{p}}$.

In view of Lemma 4.6, we will not invoke equation (4.24) to bound the function $\Theta_{\mathfrak{p}}$. Instead, we will take advantage of the regularizing properties of equation (4.15), and rely on the initial estimates of Lemma 4.5, to bound the $L^{q}$-norm of $N_{\mathfrak{p}}$ (and actually, its first order derivatives) independently on $\mathfrak{p}$. We will then deduce from (4.25) and (4.26), $L^{q}$-estimates of some low order derivatives of $\Theta_{\mathfrak{p}}$. This in turn will provide new bounds on the nonlinear terms $\mathcal{R}_{\varepsilon_{\mathfrak{p}}}^{i, j}$, and on their first order derivatives, improving the estimates of Lemma 4.5. Using in particular, the inductive nature of (4.26), we will iterate the argument to obtain $L^{q}$-bounds on any order derivatives of $N_{\mathfrak{p}}$ and $\Theta_{\mathfrak{p}}$, and complete the proof of Proposition 3 (see Section 6 below). Notice that this strategy will first require to analyse the regularizing nature of (4.15) which becomes more transparent taking its Fourier transform.

4.4. Kernels of the rescaled equations. We derive a new formulation of (4.15) which brings out its regularizing properties. Taking the Fourier transform of the previous rescaled equations, we deduce 
Corollary 4.1. Let $v$ be a finite energy solution to (TWc) on $\mathbb{R}^{2}$ satisfying (3.6), and let $N$ and $\Theta$ be the corresponding functions defined by (4.1). Then, $\widehat{N}$ and $\widehat{\Theta}$ satisfy

$$
\begin{gathered}
\left(1+\frac{\varepsilon^{2}}{2} \xi_{1}^{2}+\frac{\varepsilon^{4}}{4} \xi_{2}^{2}\right) \widehat{N}(\xi)-i \sqrt{1-\frac{\varepsilon^{2}}{2}} \xi_{1} \widehat{\Theta}(\xi)=\varepsilon^{2} \widehat{\mathcal{R}_{\varepsilon, 1}}(\xi), \\
\left(\xi_{1}^{2}+\frac{\varepsilon^{2}}{2} \xi_{2}^{2}\right) \widehat{\Theta}(\xi)+i \sqrt{1-\frac{\varepsilon^{2}}{2}} \xi_{1} \widehat{N}(\xi)=\varepsilon^{2} \widehat{\mathcal{R}_{\varepsilon, 2}}(\xi),
\end{gathered}
$$

and

$$
\left(\xi_{1}^{4}+|\xi|^{2}+\varepsilon^{2} \xi_{1}^{2} \xi_{2}^{2}+\frac{\varepsilon^{4}}{4} \xi_{2}^{4}\right) \widehat{N}(\xi)=\xi_{1}^{2}\left(\frac{1}{3} \widehat{N^{2}}(\xi)+\frac{1}{6} \widehat{\left(\partial_{1} \Theta\right)^{2}}(\xi)\right)+\varepsilon^{2} \widehat{\mathcal{R}_{\varepsilon}}(\xi) .
$$

Proof. Equations (4.29), (4.30) and (4.31) follow from taking the Fourier transform of equations (4.13), (4.14) and (4.15).

At this stage, it is presumably worthwhile to compare equations (4.31) and (2.2). This leads us to consider the perturbed kernel $K_{\varepsilon}$, whose Fourier transform is given by

$$
\widehat{K}_{\varepsilon}(\xi)=\frac{\xi_{1}^{2}}{|\xi|^{2}+\xi_{1}^{4}+\varepsilon^{2} \xi_{1}^{2} \xi_{2}^{2}+\frac{\varepsilon^{4}}{4} \xi_{2}^{4}} .
$$

The kernel $K_{\varepsilon}$ is a regularization of the kernel $K_{0}$, since it belongs to $H^{\frac{1}{4}}\left(\mathbb{R}^{2}\right)$ (see Lemma 5.1 below), and tends to $K_{0}$ in $L^{2}\left(\mathbb{R}^{2}\right)$, as $\varepsilon \rightarrow 0$, by the dominated convergence theorem. We will extensively use this additional regularizing property of $K_{\varepsilon}$ to compute estimates of the function $N$.

More generally, since

$$
\widehat{\mathcal{R}_{\varepsilon}}(\xi)=-\sum_{i+j=2} \xi_{i} \xi_{j} \widehat{\mathcal{R}}_{\varepsilon}^{i, j}(\xi),
$$

we also introduce the kernels $K_{\varepsilon}^{i, j}$ defined by

$$
\widehat{K_{\varepsilon}^{i, j}}(\xi)=\frac{\xi_{1}^{i} \xi_{2}^{j}}{|\xi|^{2}+\xi_{1}^{4}+\varepsilon^{2} \xi_{1}^{2} \xi_{2}^{2}+\frac{\varepsilon^{4}}{4} \xi_{2}^{4}},
$$

for any $0 \leq i, j \leq 4$ such that $2 \leq i+j \leq 4$ (so that, in particular, $K_{\varepsilon}=K_{\varepsilon}^{2,0}$ ). We then recast equation (4.15) as a convolution equation

$$
N_{\mathfrak{p}}=K_{\varepsilon_{\mathfrak{p}}}^{2,0} \star f_{\mathfrak{p}}-\sum_{i+j=2} \varepsilon_{\mathfrak{p}}^{2} K_{\varepsilon_{\mathfrak{p}}}^{i, j} \star \mathcal{R}_{\varepsilon_{\mathfrak{p}}}^{i, j}
$$

where

$$
f_{\mathfrak{p}}=\frac{1}{3} N_{\mathfrak{p}}^{2}+\frac{1}{6}\left(\partial_{1} \Theta_{\mathfrak{p}}\right)^{2} .
$$

In view of the multiplier properties of the kernels $K_{\varepsilon_{\mathfrak{p}}}^{i, j}$ (see Lemma 5.2 below), equation (4.33) provides a control on the $L^{q}$-norm of $N_{\mathfrak{p}}$ in function of the $L^{q_{-}}$ norms of the nonlinear terms $f_{\mathfrak{p}}$ and $\mathcal{R}_{\varepsilon_{\mathfrak{p}}}^{i, j}$. This control is the starting point of the proof of Proposition 3, which follows combining the superlinear nature of the nonlinear terms $f_{\mathfrak{p}}$ and $\mathcal{R}_{\varepsilon_{\mathfrak{p}}}^{i, j}$ with the estimates of $\Theta_{\mathfrak{p}}$ provided by Lemma 4.6 (see Section 6 below). 


\section{Properties of the kernels $K_{\varepsilon}^{i, j}$}

We now turn to the analysis of the kernels $K_{\varepsilon}^{i, j}$. In particular, we provide a number of estimates, which are required by the proof of Proposition 3.

5.1. $H^{\alpha}$-estimates of the kernels. For given $0 \leq \alpha<1$, we establish $H^{\alpha}-$ estimates for the kernels $K_{\varepsilon}^{i, j}$. We first consider their $\dot{H}^{\alpha}$-semi-norms defined in the Fourier space by

$$
\left\|K_{\varepsilon}^{i, j}\right\|_{\dot{H}^{\alpha}\left(\mathbb{R}^{2}\right)}^{2}=\int_{\mathbb{R}^{2}}|\xi|^{2 \alpha}\left|\widehat{K_{\varepsilon}^{i, j}}(\xi)\right|^{2} d \xi .
$$

Lemma 5.1. Let $0<\varepsilon \leq 1$ and $0 \leq \alpha<1$. Then,

$$
\left\|K_{\varepsilon}^{2,0}\right\|_{\dot{H}^{\alpha}\left(\mathbb{R}^{2}\right)} \leq K(\alpha)\left(1+\varepsilon^{\frac{1}{2}-2 \alpha}\right),\left\|K_{\varepsilon}^{1,1}\right\|_{\dot{H}^{\alpha}\left(\mathbb{R}^{2}\right)} \leq K(\alpha)\left(1+\varepsilon^{-\frac{1}{2}-2 \alpha}\right),
$$

and

$$
\left\|K_{\varepsilon}^{0,2}\right\|_{\dot{H}^{\alpha}\left(\mathbb{R}^{2}\right)} \leq K(\alpha)\left(1+\varepsilon^{-\frac{3}{2}-2 \alpha}\right) .
$$

Proof. The proof is an explicit computation. In view of the definition of the semi-norms, we compute using polar coordinates, and noticing that $i+j=2$,

$$
\begin{aligned}
& \left\|K_{\varepsilon}^{i, j}\right\|_{\dot{H}^{\alpha}\left(\mathbb{R}^{2}\right)}^{2}=\int_{\mathbb{R}^{2}} \frac{|\xi|^{2 \alpha} \xi_{1}^{2 i} \xi_{2}^{2 j}}{\left(|\xi|^{2}+\xi_{1}^{4}+\varepsilon^{2} \xi_{1}^{2} \xi_{2}^{2}+\frac{\varepsilon^{4}}{4} \xi_{2}^{4}\right)^{2}} d \xi \\
& =\int_{0}^{+\infty} \int_{0}^{2 \pi} r^{2 \alpha+1} \frac{\cos (\theta)^{2 i} \sin (\theta)^{2 j}}{\left(1+r^{2} \cos (\theta)^{4}+\varepsilon^{2} r^{2} \cos (\theta)^{2} \sin (\theta)^{2}+\frac{\varepsilon^{4}}{4} r^{2} \sin (\theta)^{4}\right)^{2}} d r d \theta \\
& =4 \int_{0}^{+\infty} \int_{0}^{+\infty} r^{2 \alpha+1} \frac{u^{2 j}\left(1+u^{2}\right)^{3-i-j}}{\left(\left(1+u^{2}\right)^{2}+r^{2}+\varepsilon^{2} r^{2} u^{2}+\frac{\varepsilon^{4}}{4} r^{2} u^{4}\right)^{2}} d r d u,
\end{aligned}
$$

where we have set $u=\tan (\theta)$ in the last integral. The previous computation leads us to introduce the quantity

$$
J_{\beta, \varepsilon}(r)=\int_{0}^{+\infty} \frac{u^{2 \beta}}{\left(\left(1+u^{2}\right)^{2}+r^{2}+\varepsilon^{2} r^{2} u^{2}+\frac{\varepsilon^{4}}{4} r^{2} u^{4}\right)^{2}} d u,
$$

so that

$$
\left\|K_{\varepsilon}^{i, j}\right\|_{\dot{H}^{\alpha}\left(\mathbb{R}^{2}\right)}^{2} \leq K \int_{0}^{+\infty} r^{2 \alpha+1}\left(J_{\beta_{1}, \varepsilon}(r)+J_{\beta_{2}, \varepsilon}(r)\right) d r,
$$

where $\beta_{1}=j$ and $\beta_{2}=3-i$. We now claim that

$$
\int_{0}^{+\infty} r^{2 \alpha+1} J_{\beta, \varepsilon}(r) d r \leq K(\alpha, \beta)\left(1+\frac{1}{\varepsilon^{4 \alpha+2 \beta-3}}\right),
$$

for any $0 \leq \beta<\frac{7}{2}$ and any $0 \leq \alpha<1$. We postpone the proof of Claim (5.4), and first complete the proof of Lemma 5.1. Combining identity (5.3) with (5.4), we obtain

$$
\left\|K_{\varepsilon}^{i, j}\right\|_{\dot{H}^{\alpha}\left(\mathbb{R}^{2}\right)}^{2} \leq K\left(1+\frac{1}{\varepsilon^{4 \alpha+2 j-3}}+\frac{1}{\varepsilon^{4 \alpha+3-2 i}}\right),
$$

and the conclusion follows applying this inequality for the various choices of $i$ and $j$. 
Proof of Claim (5.4). In order to estimate the integral in the left-hand side of Claim (5.4), we first compute some bounds for the function $J_{\beta, \varepsilon}$. When $0 \leq r \leq 1$, we have

$$
\left|J_{\beta, \varepsilon}(r)\right| \leq \int_{0}^{+\infty} \frac{u^{2 \beta}}{1+u^{8}} d u \leq K(\beta),
$$

since $0 \leq \beta<\frac{7}{2}$. On the other hand, when $r>1$, we compute

$$
\left|J_{\beta, \varepsilon}(r)\right| \leq K\left(\int_{0}^{1} \frac{d u}{1+r^{4}}+\int_{1}^{\frac{1}{\varepsilon}} \frac{u^{2 \beta}}{\left(u^{4}+r^{2}\right)^{2}} d u+\int_{\frac{1}{\varepsilon}}^{+\infty} \frac{u^{2 \beta-8}}{\left(1+r^{2} \varepsilon^{4}\right)^{2}} d u\right),
$$

so that, since $0 \leq \beta<\frac{7}{2}$,

$$
\left|J_{\beta, \varepsilon}(r)\right| \leq K(\beta)\left(\frac{1}{r^{4}}+r^{\beta-\frac{7}{2}}+\varepsilon^{7-2 \beta}\right),
$$

when $1 \leq r \leq \frac{1}{\varepsilon^{2}}$. Similarly, when $r \geq \frac{1}{\varepsilon^{2}}$,

$$
\left|J_{\beta, \varepsilon}(r)\right| \leq K(\beta)\left(\frac{1}{r^{4}}+\frac{1}{\varepsilon^{2 \beta+1} r^{4}}+\frac{\varepsilon^{7-2 \beta}}{\left(1+r^{2} \varepsilon^{4}\right)^{2}}\right) .
$$

Estimates (5.5), (5.6) and (5.7) finally provide Claim (5.4), when $0 \leq \alpha<1$.

Since inequalities (5.1) and (5.2) are also valid for $\alpha=0$, i.e. for the $L^{2}$-norm, we may remove the dots in inequalities (5.1) and (5.2). Notice in particular that we have the bounds

$$
\left\|K_{\varepsilon}^{1,1}\right\|_{H^{\alpha}\left(\mathbb{R}^{2}\right)}+\varepsilon\left\|K_{\varepsilon}^{1,2}\right\|_{H^{\alpha}\left(\mathbb{R}^{2}\right)}+\varepsilon^{2}\left\|K_{\varepsilon}^{2,2}\right\|_{H^{\alpha}\left(\mathbb{R}^{2}\right)} \leq K(\alpha),
$$

for any $0 \leq \alpha \leq \frac{1}{4}$.

5.2. Multiplier properties of the kernels. We now provide some multiplier properties of the kernels $K_{\varepsilon}^{i, j}$. Our analysis relies on a theorem by Lizorkin [26] ${ }^{1}$ , which we first recall for sake of completeness.

THEOREM 5.1 ([26]). Let $\widehat{K}$ be a bounded function in $\mathcal{C}^{2}\left(\mathbb{R}^{2} \backslash\{0\}\right)$, and assume that

$$
\xi_{1}^{k_{1}} \xi_{2}^{k_{2}} \partial_{1}^{k_{1}} \partial_{2}^{k_{2}} \widehat{K}(\xi) \in L^{\infty}\left(\mathbb{R}^{2}\right)
$$

for any $0 \leq k_{1}, k_{2} \leq 1$ such that $k_{1}+k_{2} \leq 2$. Then, $\widehat{K}$ is a multiplier from $L^{q}\left(\mathbb{R}^{2}\right)$ to $L^{q}\left(\mathbb{R}^{2}\right)$ for any $1<q<+\infty$. More precisely, given any $1<q<+\infty$, there exists a constant $K(q)$, depending only on $q$, such that

$$
\|K \star f\|_{L^{q}\left(\mathbb{R}^{2}\right)} \leq K(q) M(\widehat{K})\|f\|_{L^{q}\left(\mathbb{R}^{2}\right)}, \forall f \in L^{q}\left(\mathbb{R}^{2}\right),
$$

where we denote

$M(\widehat{K}) \equiv \sup \left\{\left|\xi_{1}\right|^{k_{1}}\left|\xi_{2}\right|^{k_{2}}\left|\partial_{1}^{k_{1}} \partial_{2}^{k_{2}} \widehat{K}(\xi)\right|, \xi \in \mathbb{R}^{2}, 0 \leq k_{1} \leq 1,0 \leq k_{2} \leq 1, k_{1}+k_{2} \leq 2\right\}$.

Applying Theorem 5.1 to the kernels $K_{\varepsilon}^{i, j}$, we obtain

\footnotetext{
${ }^{1}$ Estimate (5.9) in Theorem 5.1 is more precisely a consequence of Lemma 6 and of the proof of Theorem 8 in $[\mathbf{2 6}]$.
} 
LEMMA 5.2. Let $1<q<+\infty$. Given any integers $0 \leq i, j \leq 4$ such that $2 \leq i+j \leq 4$, we denote

$$
\kappa_{i, j}=\max \{i+2 j-4,0\},
$$

Then, there exists some positive constant $K(q)$, not depending on $\varepsilon$, such that

$$
\left\|K_{\varepsilon}^{i, j} \star f\right\|_{L^{q}\left(\mathbb{R}^{2}\right)} \leq \frac{K(q)}{\varepsilon^{\kappa_{i, j}}}\|f\|_{L^{q}\left(\mathbb{R}^{2}\right)},
$$

for any function $f \in L^{q}\left(\mathbb{R}^{2}\right)$ and any $\varepsilon>0$.

PROOF. Inequality (5.10) is a consequence of (5.9) once we have checked that the functions $\widehat{K_{\varepsilon}^{i, j}}$ satisfy the assumptions of Theorem 5.1, and established the dependence with respect to $\varepsilon$ of the quantity $M\left(\widehat{K_{\varepsilon}^{i, j}}\right)$.

First notice that the functions $\widehat{K_{\varepsilon}^{i, j}}$, which are bounded on $\mathbb{R}^{2}$, and belong to $\mathcal{C}^{2}\left(\mathbb{R}^{2} \backslash\{0\}\right)$, may be written as

$$
\widehat{K_{\varepsilon}^{i, j}}(\xi)=\frac{\xi_{1}^{i} \xi_{2}^{j}}{Q(\xi)},
$$

where $Q(\xi) \equiv|\xi|^{2}+\xi_{1}^{4}+\varepsilon^{2} \xi_{1}^{2} \xi_{2}^{2}+\frac{\varepsilon^{4}}{4} \xi_{2}^{4}$. We therefore compute

$$
\begin{aligned}
& \xi_{1} \partial_{1} \widehat{K_{\varepsilon}^{i, j}}(\xi)=i \frac{\xi_{1}^{i} \xi_{2}^{j}}{Q(\xi)}-\frac{\xi_{1}^{i} \xi_{2}^{j}}{Q(\xi)} \frac{\xi_{1} \partial_{1} Q(\xi)}{Q(\xi)} \\
& \xi_{2} \partial_{2} \widehat{K_{\varepsilon}^{i, j}}(\xi)=j \frac{\xi_{1}^{i} \xi_{2}^{j}}{Q(\xi)}-\frac{\xi_{1}^{i} \xi_{2}^{j}}{Q(\xi)} \frac{\xi_{2} \partial_{2} Q(\xi)}{Q(\xi)}
\end{aligned}
$$

and

$$
\begin{aligned}
\xi_{1} \xi_{2} \partial_{1} \partial_{2} \widehat{K_{\varepsilon}^{i, j}}(\xi)= & \frac{\xi_{1}^{i} \xi_{2}^{j}}{Q(\xi)}\left(i j-(i+j) \frac{\xi_{1} \partial_{1} Q(\xi)+\xi_{2} \partial_{2} Q(\xi)}{Q(\xi)}\right. \\
& \left.-\frac{\xi_{1} \xi_{2} \partial_{1} \partial_{2} Q(\xi)}{Q(\xi)}+2 \frac{\xi_{1} \partial_{1} Q(\xi)}{Q(\xi)} \frac{\xi_{2} \partial_{2} Q(\xi)}{Q(\xi)}\right)
\end{aligned}
$$

On the other hand, we check that

$$
\varepsilon^{\kappa_{i, j}}\left|\xi_{1}\right|^{i}\left|\xi_{2}\right|^{j} \leq 4 Q(\xi),\left|\xi_{k}\right|\left|\partial_{k} Q(\xi)\right| \leq 4 Q(\xi) \text {, and }\left|\xi_{1}\right|\left|\xi_{2}\right|\left|\partial_{1} \partial_{2} Q(\xi)\right| \leq 4 Q(\xi),
$$

so that, by (5.11) and (5.12), there exists some universal constant $K$ such that

$$
\varepsilon^{\kappa_{i, j}} M\left(\widehat{K_{\varepsilon}^{i, j}}\right) \leq K
$$

Inequality (5.10) then follows from (5.9) applying Theorem 5.1.

\section{Sobolev bounds for $N_{\mathfrak{p}}$ and $\Theta_{\mathfrak{p}}$}

This section is devoted to the proof of the Sobolev estimates of $N_{\mathfrak{p}}, \partial_{1} \Theta_{\mathfrak{p}}$ and $\partial_{2} \Theta_{\mathfrak{p}}$ stated in Proposition 3. As previously mentioned in Section 4, we focus on Sobolev bounds on $N_{\mathfrak{p}}$.

Proposition 6.1. Let $\alpha \in \mathbb{N}^{2}$ and $1<q<+\infty$. There exists some constant $K(q, \alpha)$, depending possibly on $\alpha$ and $q$, but not on $\mathfrak{p}$, such that

$$
\begin{aligned}
& \left\|\partial^{\alpha} N_{\mathfrak{p}}\right\|_{L^{q}\left(\mathbb{R}^{2}\right)}+\left\|\partial_{1} \partial^{\alpha} N_{\mathfrak{p}}\right\|_{L^{q}\left(\mathbb{R}^{2}\right)}+\left\|\partial_{2} \partial^{\alpha} N_{\mathfrak{p}}\right\|_{L^{q}\left(\mathbb{R}^{2}\right)} \\
& +\left\|\partial_{1}^{2} \partial^{\alpha} N_{\mathfrak{p}}\right\|_{L^{q}\left(\mathbb{R}^{2}\right)}+\varepsilon_{\mathfrak{p}}\left\|\partial_{1} \partial_{2} \partial^{\alpha} N_{\mathfrak{p}}\right\|_{L^{q}\left(\mathbb{R}^{2}\right)}+\varepsilon_{\mathfrak{p}}^{2}\left\|\partial_{2}^{2} \partial^{\alpha} N_{\mathfrak{p}}\right\|_{L^{q}\left(\mathbb{R}^{2}\right)} \leq K(q, \alpha),
\end{aligned}
$$

for any $\mathfrak{p}$ sufficiently small. 
REMARK 6.1. The proof of Proposition 6.1 is by induction on the derivation order $\alpha$. The inductive assumption is given by (6.1). This explains the redundant form of this inequality.

Proposition 3 is a direct consequence of Proposition 6.1 invoking rescaled inequalities (4.25) and (4.26) to bound the functions $\partial_{1} \Theta_{\mathfrak{p}}$ and $\partial_{2} \Theta_{\mathfrak{p}}$.

Proof of Proposition 3 (assuming Proposition 6.1). In view of (6.1), given any $k \in \mathbb{N}$ and any $1<q<+\infty$, there exists some positive constant $K(k, q)$, not depending on $\mathfrak{p}$, such that

$$
\left\|N_{\mathfrak{p}}\right\|_{W^{k, q}\left(\mathbb{R}^{2}\right)} \leq K(k, q),
$$

for any $\mathfrak{p}$ sufficiently small. In particular, by Sobolev embedding theorem,

$$
\left\|N_{\mathfrak{p}}\right\|_{\mathcal{C}^{k}\left(\mathbb{R}^{2}\right)} \leq K(k) .
$$

Using (6.2) and (6.3), inequality (4.26) becomes

$$
\Xi_{\mathfrak{p}}(q, \alpha) \leq K(q, \alpha)\left(1+\varepsilon_{\mathfrak{p}}^{2} \sum_{0 \leq \beta<\alpha} \Xi_{\mathfrak{p}}(q, \alpha-\beta)\right),
$$

where we have set as in Lemma 4.6,

$$
\Xi_{\mathfrak{p}}(q, \alpha) \equiv\left\|\partial^{\alpha} \partial_{1} \Theta_{\mathfrak{p}}\right\|_{L^{q}\left(\mathbb{R}^{2}\right)}+\varepsilon_{\mathfrak{p}}\left\|\partial^{\alpha} \partial_{2} \Theta_{\mathfrak{p}}\right\|_{L^{q}\left(\mathbb{R}^{2}\right)} .
$$

By $(4.25)$ and $(6.2)$, the quantity $\Xi_{\mathfrak{p}}(q,(0,0))$ is bounded independently on $\mathfrak{p}$, so that it follows by induction from formula (6.4) that $\Xi_{\mathfrak{p}}(q, \alpha)$ is bounded independently on $\mathfrak{p}$ for any $1<q<+\infty$ and any $\alpha \in \mathbb{N}^{2}$. Inequality (11) follows invoking Sobolev embedding theorem for $q=+\infty$. This completes the proof of Proposition 3.

The remainder of this section is devoted to the proof of Proposition 6.1. As previously mentioned in Subsection 4.4, the proof relies on decomposition (4.33). Recall that it is proved in [16] that the functions $\eta$ and $\varphi$, and therefore $N_{\mathfrak{p}}$ and $\Theta_{\mathfrak{p}}$, belong to $W^{k, q}\left(\mathbb{R}^{2}\right)$ for any $k \in \mathbb{N}$ and any $1<q \leq+\infty$. Hence, we can differentiate (4.33) to any order $\alpha \in \mathbb{N}^{2}$ to obtain

$$
\partial^{\alpha} N_{\mathfrak{p}}=K_{\varepsilon_{\mathfrak{p}}}^{2,0} \star \partial^{\alpha} f_{\mathfrak{p}}+\varepsilon_{\mathfrak{p}}^{2} \sum_{i+j=2} K_{\varepsilon_{\mathfrak{p}}}^{i, j} \star \partial^{\alpha} \mathcal{R}_{\varepsilon_{\mathfrak{p}}}^{i, j}
$$

Taking the $L^{q}$-norm of this expression and invoking the regularizing properties of the kernels provided by Lemma 5.2, we are led to

$$
\left\|\partial^{\alpha} N_{\mathfrak{p}}\right\|_{L^{q}\left(\mathbb{R}^{2}\right)} \leq K(q)\left(\left\|\partial^{\alpha} f_{\mathfrak{p}}\right\|_{L^{q}\left(\mathbb{R}^{2}\right)}+\varepsilon_{\mathfrak{p}}^{2} \sum_{i+j=2}\left\|\partial^{\alpha} \mathcal{R}_{\varepsilon_{\mathfrak{p}}}^{i, j}\right\|_{L^{q}\left(\mathbb{R}^{2}\right)}\right) .
$$

In view of definitions (4.16), (4.17), (4.18) and (4.34), the derivatives $\partial^{\alpha} f_{\mathfrak{p}}$ and $\partial^{\alpha} \mathcal{R}_{\varepsilon_{\mathfrak{p}}}^{i, j}$ in the right-hand side of (6.6) are nonlinear functions of the derivatives of $N_{\mathfrak{p}}$ and $\Theta_{\mathfrak{p}}$, so that we may estimate their $L^{q}$-norms using Sobolev bounds on $N_{\mathfrak{p}}$ and $\Theta_{p}$.

This provides an iterative scheme to estimate the Sobolev norms of $N_{\mathfrak{p}}$. Using the available information on the nonlinear source terms $f_{\mathfrak{p}}$ and $\mathcal{R}_{\varepsilon_{\mathfrak{p}}}^{i, j}$, which is initially reduced to Lemma 4.5 , we improve the regularity and integrability properties of $N_{\mathfrak{p}}$ using inequality (6.6). This in turn provides improved bounds of the nonlinear terms $f_{\mathfrak{p}}$ and $\mathcal{R}_{\varepsilon_{\mathfrak{p}}}^{i, j}$.

As a consequence, we prove (6.1) by induction on the derivation order $\alpha$. We first compute $L^{q}$-estimates of the nonlinear terms $f_{\mathfrak{p}}$ and $\mathcal{R}_{\varepsilon_{\mathfrak{p}}}^{i, j}$, and of convolution 
equation (4.33). In particular, this requires to bound some derivatives of the phase $\Theta_{\mathfrak{p}}$, which is made possible invoking Lemma 4.6. Using the initial bounds given by Lemma 4.5, we conclude that inequality (6.1) holds for $\alpha=(0,0)$. We then turn to higher order estimates. Assuming that (6.1) holds for any index $\alpha$ such that $|\alpha| \leq k$, we derive $L^{q}$-estimates of the derivatives of order $k+1$ of the functions $f_{\mathfrak{p}}$ and $\mathcal{R}_{\varepsilon_{\mathfrak{p}}}^{i, j}$. In view of (6.6), this provides bounds for the derivatives of order $k+1$ of $N_{\mathfrak{p}}$, so that we can prove that (6.1) is also valid for any index $\alpha$ such that $|\alpha|=k+1$. This completes the sketch of the proof of Proposition 6.1 , which is detailed below.

6.1. $L^{q}$-estimates of nonlinear terms. We first compute $L^{q}$-estimates on the nonlinear terms $f_{\mathfrak{p}}, R_{\varepsilon_{\mathfrak{p}}}^{i, j}$ and $\nu_{\varepsilon_{\mathfrak{p}}}^{i, j}$.

Lemma 6.1. Let $1 \leq q<+\infty$. There exists some universal constant $K$ such that

$$
\begin{aligned}
& \left\|f_{\mathfrak{p}}\right\|_{L^{q}\left(\mathbb{R}^{2}\right)}+\left\|R_{\varepsilon_{\mathfrak{p}}}^{0,2}\right\|_{L^{q}\left(\mathbb{R}^{2}\right)}+\varepsilon_{\mathfrak{p}}\left\|R_{\varepsilon_{\mathfrak{p}}}^{1,1}\right\|_{L^{q}\left(\mathbb{R}^{2}\right)} \leq K\left\|N_{\mathfrak{p}}\right\|_{L^{2 q}\left(\mathbb{R}^{2}\right)}^{2}, \\
& \left\|R_{\varepsilon_{\mathfrak{p}}}^{2,0}\right\|_{L^{q}\left(\mathbb{R}^{2}\right)} \leq K\left(\varepsilon_{\mathfrak{p}}^{-2}\left\|N_{\mathfrak{p}}\right\|_{L^{2 q}\left(\mathbb{R}^{2}\right)}^{2}+\left\|N_{\mathfrak{p}}\right\|_{L^{3 q}\left(\mathbb{R}^{2}\right)}^{3}+\left\|\partial_{1} N_{\mathfrak{p}}\right\|_{L^{2 q}\left(\mathbb{R}^{2}\right)}^{2}\right), \\
& \left\|\nu_{\varepsilon_{\mathfrak{p}}}^{2,0}\right\|_{L^{q}\left(\mathbb{R}^{2}\right)} \leq K\left(\varepsilon_{\mathfrak{p}}^{-2}\left\|N_{\mathfrak{p}}\right\|_{L^{3 q}\left(\mathbb{R}^{2}\right)}^{3}+\left\|\partial_{2} N_{\mathfrak{p}}\right\|_{L^{2 q}\left(\mathbb{R}^{2}\right)}^{2}\right),
\end{aligned}
$$

and

$\left\|\nu_{\varepsilon_{\mathfrak{p}}}^{0,2}\right\|_{L^{q}\left(\mathbb{R}^{2}\right)} \leq K\left(\varepsilon_{\mathfrak{p}}^{-2}\left\|N_{\mathfrak{p}}\right\|_{L^{2 q}\left(\mathbb{R}^{2}\right)}^{2}+\left\|N_{\mathfrak{p}}\right\|_{L^{3 q}\left(\mathbb{R}^{2}\right)}^{3}+\left\|\partial_{1} N_{\mathfrak{p}}\right\|_{L^{2 q}\left(\mathbb{R}^{2}\right)}^{2}+\varepsilon_{\mathfrak{p}}^{2}\left\|\partial_{2} N_{\mathfrak{p}}\right\|_{L^{2 q}\left(\mathbb{R}^{2}\right)}^{2}\right)$.

Proof. Bounds (6.7), (6.8), (6.9) and (6.10) are consequences of inequalities (4.19), (4.20) and (4.21) using Hölder inequalities. For the quantities involving the functions $\partial_{1} \Theta_{\mathfrak{p}}$ and $\partial_{2} \Theta_{\mathfrak{p}}$, we also use (4.25) to compute

$$
\left\|\left(\partial_{1} \Theta_{\mathfrak{p}}\right)^{2}\right\|_{L^{q}\left(\mathbb{R}^{2}\right)}+\varepsilon_{\mathfrak{p}}\left\|N_{\mathfrak{p}} \partial_{2} \Theta_{\mathfrak{p}}\right\|_{L^{q}\left(\mathbb{R}^{2}\right)}+\varepsilon_{\mathfrak{p}}^{2}\left\|\left(\partial_{2} \Theta_{\mathfrak{p}}\right)^{2}\right\|_{L^{q}\left(\mathbb{R}^{2}\right)} \leq K(q)\left\|N_{\mathfrak{p}}\right\|_{L^{2 q}\left(\mathbb{R}^{2}\right)}^{2},
$$

whereas

$$
\begin{gathered}
\left\|N_{\mathfrak{p}}\left(\partial_{1} \Theta_{\mathfrak{p}}\right)^{2}\right\|_{L^{q}\left(\mathbb{R}^{2}\right)}+\varepsilon_{\mathfrak{p}}^{2}\left\|N_{\mathfrak{p}}\left(\partial_{2} \Theta_{\mathfrak{p}}\right)^{2}\right\|_{L^{q}\left(\mathbb{R}^{2}\right)} \\
\leq K(q)\left\|N_{\mathfrak{p}}\right\|_{L^{3 q}\left(\mathbb{R}^{2}\right)}\left(\left\|\partial_{1} \Theta_{\mathfrak{p}}\right\|_{L^{3 q}\left(\mathbb{R}^{2}\right)}^{2}+\varepsilon_{\mathfrak{p}}^{2}\left\|\partial_{2} \Theta_{\mathfrak{p}}\right\|_{L^{3 q}\left(\mathbb{R}^{2}\right)}^{2}\right) \leq K(q)\left\|N_{\mathfrak{p}}\right\|_{L^{3 q}\left(\mathbb{R}^{2}\right)}^{3} .
\end{gathered}
$$

6.2. $L^{q}$-estimates of the convolution equation. We now compute $L^{q}$ estimates of equation (4.33) invoking the multiplier properties of the kernels $K_{\varepsilon}^{i, j}$ given by Lemma 5.2 , and the previous $L^{q}$-estimates on the nonlinear terms $f_{\mathfrak{p}}, R_{\varepsilon_{\mathfrak{p}}}^{i, j}$ and $\nu_{\varepsilon_{\mathfrak{p}}}^{i, j}$. This provides

Lemma 6.2. Let $1<q<+\infty$. There exists some constant $K(q)$, depending only on $q$, such that

$$
\begin{gathered}
\left\|N_{\mathfrak{p}}\right\|_{L^{q}\left(\mathbb{R}^{2}\right)}+\left\|\partial_{1} N_{\mathfrak{p}}\right\|_{L^{q}\left(\mathbb{R}^{2}\right)}+\left\|\partial_{2} N_{\mathfrak{p}}\right\|_{L^{q}\left(\mathbb{R}^{2}\right)} \\
+\left\|\partial_{1}^{2} N_{\mathfrak{p}}\right\|_{L^{q}\left(\mathbb{R}^{2}\right)}+\varepsilon_{\mathfrak{p}}\left\|\partial_{1} \partial_{2} N_{\mathfrak{p}}\right\|_{L^{q}\left(\mathbb{R}^{2}\right)}+\varepsilon_{\mathfrak{p}}^{2}\left\|\partial_{2}^{2} N_{\mathfrak{p}}\right\|_{L^{q}\left(\mathbb{R}^{2}\right)} \\
\leq K(q)\left(\left\|N_{\mathfrak{p}}\right\|_{L^{2 q}\left(\mathbb{R}^{2}\right)}^{2}+\varepsilon_{\mathfrak{p}}^{2}\left\|N_{\mathfrak{p}}\right\|_{L^{3 q}\left(\mathbb{R}^{2}\right)}^{3}+\varepsilon_{\mathfrak{p}}^{2}\left\|\partial_{1} N_{\mathfrak{p}}\right\|_{L^{2 q}\left(\mathbb{R}^{2}\right)}^{2}+\varepsilon_{\mathfrak{p}}^{4}\left\|\partial_{2} N_{\mathfrak{p}}\right\|_{L^{2 q}\left(\mathbb{R}^{2}\right)}^{2}\right),
\end{gathered}
$$

for any $\mathfrak{p}$ sufficiently small. 
Proof. Given any $\alpha=\left(\alpha_{1}, \alpha_{2}\right)$ such that $0 \leq \alpha_{1}+\alpha_{2} \leq 2$, we estimate the $L^{q}$-norm of $\partial^{\alpha} N_{\mathfrak{p}}$ using equations (4.33), so that

$$
\begin{aligned}
\left\|\partial^{\alpha} N_{\mathfrak{p}}\right\|_{L^{q}\left(\mathbb{R}^{2}\right)} & \leq\left\|\partial^{\alpha} K_{\varepsilon_{\mathfrak{p}}}^{2,0} \star f_{\mathfrak{p}}\right\|_{L^{q}\left(\mathbb{R}^{2}\right)}+\varepsilon_{\mathfrak{p}}^{2} \sum_{i+j=2}\left\|\partial^{\alpha} K_{\varepsilon_{\mathfrak{p}}}^{i, j} \star R_{\varepsilon_{\mathfrak{p}}}^{i, j}\right\|_{L^{q}\left(\mathbb{R}^{2}\right)} \\
& +\varepsilon_{\mathfrak{p}}^{4}\left\|\partial^{\alpha} K_{\varepsilon_{\mathfrak{p}}}^{2,0} \star \nu_{\varepsilon_{\mathfrak{p}}}^{2,0}\right\|_{L^{q}\left(\mathbb{R}^{2}\right)}+\varepsilon_{\mathfrak{p}}^{4}\left\|\partial^{\alpha} K_{\varepsilon_{\mathfrak{p}}}^{0,2} \star \nu_{\varepsilon_{\mathfrak{p}}}^{0,2}\right\|_{L^{q}\left(\mathbb{R}^{2}\right)} .
\end{aligned}
$$

Since by (4.32),

$$
\partial^{\alpha} K_{\varepsilon_{\mathfrak{p}}}^{j, k}=i^{\alpha_{1}+\alpha_{2}} K_{\varepsilon_{\mathfrak{p}}}^{j+\alpha_{1}, k+\alpha_{2}},
$$

the multiplier properties of Lemma 5.2 provide

$$
\begin{gathered}
\left\|N_{\mathfrak{p}}\right\|_{L^{q}\left(\mathbb{R}^{2}\right)} \leq \quad \\
\left.+\varepsilon_{\mathfrak{p}}^{4}\left\|\nu_{\varepsilon_{\mathfrak{p}}}^{2,0}\right\|_{L^{q}\left(\mathbb{R}^{2}\right)}+\varepsilon_{\mathfrak{p}}^{4}\left\|\nu_{\varepsilon_{\mathfrak{p}}}^{0,2}\right\|_{L^{q}\left(\mathbb{R}^{2}\right)}\right), \\
\left\|\partial_{1} N_{\mathfrak{p}}\right\|_{L^{q}\left(\mathbb{R}^{2}\right)} \leq \quad K(q)\left(\left\|f_{\mathfrak{p}}\right\|_{L^{q}\left(\mathbb{R}^{2}\right)}+\varepsilon_{\mathfrak{p}}^{2}\left\|R_{\varepsilon_{\mathfrak{p}}}^{2,0}\right\|_{L^{q}\left(\mathbb{R}^{2}\right)}+\varepsilon_{\mathfrak{p}}^{2}\left\|R_{\varepsilon_{\mathfrak{p}}}^{1,1}\right\|_{L^{q}\left(\mathbb{R}^{2}\right)}\left\|R_{\varepsilon_{\mathfrak{p}}}^{i, j}\right\|_{L^{q}\left(\mathbb{R}^{2}\right)}\right. \\
\left.+\varepsilon_{\mathfrak{p}}\left\|R_{\varepsilon_{\mathfrak{p}}}^{0,2}\right\|_{L^{q}\left(\mathbb{R}^{2}\right)}+\varepsilon_{\mathfrak{p}}^{4}\left\|\nu_{\varepsilon_{\mathfrak{p}}}^{2,0}\right\|_{L^{q}\left(\mathbb{R}^{2}\right)}+\varepsilon_{\mathfrak{p}}^{3}\left\|\nu_{\varepsilon_{\mathfrak{p}}}^{0,2}\right\|_{L^{q}\left(\mathbb{R}^{2}\right)}\right),
\end{gathered}
$$

and

$$
\begin{aligned}
& \left\|\partial_{2} N_{\mathfrak{p}}\right\|_{L^{q}\left(\mathbb{R}^{2}\right)}+\left\|\partial_{1}^{2} N_{\mathfrak{p}}\right\|_{L^{q}\left(\mathbb{R}^{2}\right)}+\varepsilon_{\mathfrak{p}}\left\|\partial_{1} \partial_{2} N_{\mathfrak{p}}\right\|_{L^{q}\left(\mathbb{R}^{2}\right)}+\varepsilon_{\mathfrak{p}}^{2}\left\|\partial_{2}^{2} N_{\mathfrak{p}}\right\|_{L^{q}\left(\mathbb{R}^{2}\right)} \leq \\
& K(q)\left(\left\|f_{\mathfrak{p}}\right\|_{L^{q}\left(\mathbb{R}^{2}\right)}+\varepsilon_{\mathfrak{p}}^{2}\left\|R_{\varepsilon_{\mathfrak{p}}}^{2,0}\right\|_{L^{q}\left(\mathbb{R}^{2}\right)}+\varepsilon_{\mathfrak{p}}\left\|R_{\varepsilon_{\mathfrak{p}}}^{1,1}\right\|_{L^{q}\left(\mathbb{R}^{2}\right)}\right. \\
& \left.+\left\|R_{\varepsilon_{\mathfrak{p}}}^{0,2}\right\|_{L^{q\left(\mathbb{R}^{2}\right)}}+\varepsilon_{\mathfrak{p}}^{4}\left\|\nu_{\varepsilon_{\mathfrak{p}}}^{2,0}\right\|_{L^{q}\left(\mathbb{R}^{2}\right)}+\varepsilon_{\mathfrak{p}}^{2}\left\|\nu_{\varepsilon_{\mathfrak{p}}}^{0,2}\right\|_{L^{q}\left(\mathbb{R}^{2}\right)}\right)
\end{aligned}
$$

Estimate (6.11) follows invoking nonlinear bounds (6.7), (6.8), (6.9) and (6.10).

6.3. Initial bounds on $N_{\mathfrak{p}}$ and its first order derivatives. In view of (6.11), some preliminary $L^{q}$-bounds on $N_{\mathfrak{p}}, \partial_{1} N_{\mathfrak{p}}$ and $\partial_{2} N_{\mathfrak{p}}$ are required to inductively estimate the $L^{q}$-norms of these functions. These preliminary bounds are consequences of the uniform estimates given by (3.3), and the $L^{2}$-bounds provided by (4.7), (4.8) and (4.10).

Lemma 6.3. Let $2 \leq q \leq \frac{8}{3}$. There exists some constant $K(q)$, depending only on $q$, such that

$$
\left\|N_{\mathfrak{p}}\right\|_{L^{q}\left(\mathbb{R}^{2}\right)} \leq K(q),
$$

for any $\mathfrak{p}$ sufficiently small. Moreover, given any $\frac{8}{3}<q<8$, we have

$$
\varepsilon_{\mathfrak{p}}^{\frac{2}{3}}\left\|N_{\mathfrak{p}}\right\|_{L^{q}\left(\mathbb{R}^{2}\right)} \leq K(q),
$$

whereas, given any $2 \leq q \leq+\infty$,

$$
\left\|\partial_{1} N_{\mathfrak{p}}\right\|_{L^{q}\left(\mathbb{R}^{2}\right)}+\varepsilon_{\mathfrak{p}}\left\|\partial_{2} N_{\mathfrak{p}}\right\|_{L^{q}\left(\mathbb{R}^{2}\right)} \leq K(q) \varepsilon_{\mathfrak{p}}^{\frac{6}{q}-3} .
$$

Proof. For estimate (6.14), we have in view of (3.3),

$$
\left\|\partial_{1} N_{\mathfrak{p}}\right\|_{L^{\infty}\left(\mathbb{R}^{2}\right)} \leq \frac{K}{\varepsilon_{\mathfrak{p}}^{3}}, \text { and }\left\|\partial_{2} N_{\mathfrak{p}}\right\|_{L^{\infty}\left(\mathbb{R}^{2}\right)} \leq \frac{K}{\varepsilon_{\mathfrak{p}}^{4}},
$$

so that (6.14) is a consequence of (4.8) and (4.10) using standard interpolation between $L^{q}$-spaces. 
The proofs of (6.12) and (6.13) are more involved. The first step is to compute $H^{\alpha}$-estimates of $N_{\mathfrak{p}}$ combining equation (4.33) with $H^{\alpha}$-bounds (5.8) on the kernels.

STEP 1. Let $0 \leq \alpha \leq \frac{1}{4}$. There exists some constant $K(\alpha)$ such that

$$
\left\|N_{\mathfrak{p}}\right\|_{H^{\alpha}\left(\mathbb{R}^{2}\right)} \leq K(\alpha),
$$

for any $\mathfrak{p}$ sufficiently small. In particular, there exists some constant $K(q)$ such that (6.12) holds.

Applying Young inequality to decomposition (4.33), we have

$$
\begin{gathered}
\left\|N_{\mathfrak{p}}\right\|_{H^{\alpha}\left(\mathbb{R}^{2}\right)} \leq\left\|K_{\varepsilon_{\mathfrak{p}}, 0}^{2,0}\right\|_{H^{\alpha}\left(\mathbb{R}^{2}\right)}\left(\left\|f_{\mathfrak{p}}\right\|_{L^{1}\left(\mathbb{R}^{2}\right)}+\varepsilon_{\mathfrak{p}}^{2}\left\|R_{\varepsilon_{\mathfrak{p}}}^{2,0}\right\|_{L^{1}\left(\mathbb{R}^{2}\right)}+\varepsilon_{\mathfrak{p}}^{4}\left\|\nu_{\varepsilon_{\mathfrak{p}}}^{2,0}\right\|_{L^{1}\left(\mathbb{R}^{2}\right)}\right) \\
+\varepsilon_{\mathfrak{p}}^{2}\left\|K_{\varepsilon_{\mathfrak{p}}}^{1,1}\right\|_{H^{\alpha}\left(\mathbb{R}^{2}\right)}\left\|\mathcal{R}_{\varepsilon_{\mathfrak{p}}}^{1,1}\right\|_{L^{1}\left(\mathbb{R}^{2}\right)}+\varepsilon_{\mathfrak{p}}^{2}\left\|K_{\varepsilon_{\mathfrak{p}}}^{0,2}\right\|_{H^{\alpha}\left(\mathbb{R}^{2}\right)}\left(\left\|R_{\varepsilon_{\mathfrak{p}}}^{0,2}\right\|_{L^{1}\left(\mathbb{R}^{2}\right)}+\varepsilon_{\mathfrak{p}}^{2}\left\|\nu_{\varepsilon_{\mathfrak{p}}}^{0,2}\right\|_{L^{1}\left(\mathbb{R}^{2}\right)}\right) .
\end{gathered}
$$

Combining (5.8) with (4.7), (4.22) and (4.23), we derive (6.15), whereas (6.12) is a consequence of Sobolev embedding theorem,

$$
H^{\alpha}\left(\mathbb{R}^{2}\right) \hookrightarrow L^{q}\left(\mathbb{R}^{2}\right),
$$

for any $2 \leq q \leq \frac{2}{1-\alpha}$.

The second step is to compute uniform bounds on $N_{\mathfrak{p}}$ using Sobolev embedding theorem.

STEP 2. Let $\nu>0$. There exists some constant $K(\nu)$ such that

$$
\left\|N_{\mathfrak{p}}\right\|_{L^{\infty}\left(\mathbb{R}^{2}\right)} \leq K(\nu)\left(1+\varepsilon_{\mathfrak{p}}^{-1-\nu}\right)
$$

for any $\mathfrak{p}$ sufficiently small.

In view of (6.12) and (6.14), there exists some number $q>2$ such that

$$
\left\|N_{\mathfrak{p}}\right\|_{W^{1, q}\left(\mathbb{R}^{2}\right)} \leq K(\nu)\left(1+\varepsilon_{\mathfrak{p}}^{-1-\nu}\right) .
$$

Estimate (6.16) follows by Sobolev embedding theorem.

Combining with (6.12), and invoking standard interpolation between $L^{q}$-spaces, estimate (6.16) yields (6.13).

6.4. Proof of inductive assumption (6.1) for $\alpha=(0,0)$. We now rely on Lemma 6.2 to improve the preliminary estimates of Lemma 6.3. This gives

Lemma 6.4. Let $1<q<+\infty$. Then, assumption (6.1) holds for $\alpha=(0,0)$, i.e. there exists some constant $K(q)$, not depending on $\mathfrak{p}$, such that

$$
\begin{gathered}
\left\|N_{\mathfrak{p}}\right\|_{L^{q}\left(\mathbb{R}^{2}\right)}+\left\|\partial_{1} N_{\mathfrak{p}}\right\|_{L^{q}\left(\mathbb{R}^{2}\right)}+\left\|\partial_{2} N_{\mathfrak{p}}\right\|_{L^{q}\left(\mathbb{R}^{2}\right)} \\
+\left\|\partial_{1}^{2} N_{\mathfrak{p}}\right\|_{L^{q}\left(\mathbb{R}^{2}\right)}+\varepsilon_{\mathfrak{p}}\left\|\partial_{1} \partial_{2} N_{\mathfrak{p}}\right\|_{L^{q}\left(\mathbb{R}^{2}\right)}+\varepsilon_{\mathfrak{p}}^{2}\left\|\partial_{2}^{2} N_{\mathfrak{p}}\right\|_{L^{q}\left(\mathbb{R}^{2}\right)} \leq K(q),
\end{gathered}
$$

for any $\mathfrak{p}$ sufficiently small.

Proof. The proof relies on some bootstrap argument. Given any $1<q \leq \frac{4}{3}$, we deduce from $(6.11),(6.12),(6.13)$ and (6.14), that

$$
\begin{gathered}
\left\|N_{\mathfrak{p}}\right\|_{L^{q}\left(\mathbb{R}^{2}\right)}+\left\|\partial_{1} N_{\mathfrak{p}}\right\|_{L^{q}\left(\mathbb{R}^{2}\right)}+\left\|\partial_{2} N_{\mathfrak{p}}\right\|_{L^{q}\left(\mathbb{R}^{2}\right)} \\
+\left\|\partial_{1}^{2} N_{\mathfrak{p}}\right\|_{L^{q}\left(\mathbb{R}^{2}\right)}+\varepsilon_{\mathfrak{p}}\left\|\partial_{1} \partial_{2} N_{\mathfrak{p}}\right\|_{L^{q}\left(\mathbb{R}^{2}\right)}+\varepsilon_{\mathfrak{p}}^{2}\left\|\partial_{2}^{2} N_{\mathfrak{p}}\right\|_{L^{q}\left(\mathbb{R}^{2}\right)} \leq K(q),
\end{gathered}
$$

so that by Sobolev embedding theorem,

$$
\left\|N_{\mathfrak{p}}\right\|_{L^{q}\left(\mathbb{R}^{2}\right)}+\varepsilon_{\mathfrak{p}}\left\|\partial_{1} N_{\mathfrak{p}}\right\|_{L^{q}\left(\mathbb{R}^{2}\right)}+\varepsilon_{\mathfrak{p}}^{2}\left\|\partial_{2} N_{\mathfrak{p}}\right\|_{L^{q}\left(\mathbb{R}^{2}\right)} \leq K(q),
$$


for any $1<q \leq 4$. Invoking (6.11) and (6.13) once more time, we are led to

$$
\begin{gathered}
\left\|N_{\mathfrak{p}}\right\|_{L^{q}\left(\mathbb{R}^{2}\right)}+\left\|\partial_{1} N_{\mathfrak{p}}\right\|_{L^{q}\left(\mathbb{R}^{2}\right)}+\left\|\partial_{2} N_{\mathfrak{p}}\right\|_{L^{q}\left(\mathbb{R}^{2}\right)} \\
+\left\|\partial_{1}^{2} N_{\mathfrak{p}}\right\|_{L^{q}\left(\mathbb{R}^{2}\right)}+\varepsilon_{\mathfrak{p}}\left\|\partial_{1} \partial_{2} N_{\mathfrak{p}}\right\|_{L^{q}\left(\mathbb{R}^{2}\right)}+\varepsilon_{\mathfrak{p}}^{2}\left\|\partial_{2}^{2} N_{\mathfrak{p}}\right\|_{L^{q}\left(\mathbb{R}^{2}\right)} \leq K(q),
\end{gathered}
$$

for any $1<q \leq 2$. In particular, we have by Sobolev embedding theorem,

$$
\left\|N_{\mathfrak{p}}\right\|_{L^{q}\left(\mathbb{R}^{2}\right)}+\varepsilon_{\mathfrak{p}}\left\|\partial_{1} N_{\mathfrak{p}}\right\|_{L^{q}\left(\mathbb{R}^{2}\right)}+\varepsilon_{\mathfrak{p}}^{2}\left\|\partial_{2} N_{\mathfrak{p}}\right\|_{L^{q}\left(\mathbb{R}^{2}\right)} \leq K(q),
$$

for any $1<q<+\infty$, so that (6.11) now yields (6.17) for any $1<q<+\infty$. This completes the proof of Lemma 6.4.

6.5. Higher order estimates of the nonlinear terms $f_{\mathfrak{p}}$ and $\mathcal{R}_{\varepsilon_{\mathfrak{p}}}^{i, j}$. We now assume that assumption (6.1) holds for any $1<q<+\infty$ and any $\alpha \in \mathbb{N}^{2}$ such that $|\alpha| \leq k$, and prove that it remains valid when $|\alpha|=k+1$. Invoking again equation (4.33), we first derive improved Sobolev bounds on the nonlinear terms $f_{\mathfrak{p}}$ and $\mathcal{R}_{\varepsilon_{\mathfrak{p}}}^{i, j}$. In view of definitions (4.16), (4.17), (4.18) and (4.34), this requires to compute $L^{q}$-bounds on the derivatives of $\Theta_{\mathfrak{p}}$. Hence, we show

LEMMA 6.5. Let $k \in \mathbb{N}$, and assume that (6.1) holds for any $1<q<+\infty$ and any $\alpha \in \mathbb{N}^{2}$ such that $|\alpha| \leq k$. Then, there exist some positive constants $K(q, \alpha)$, not depending on $\mathfrak{p}$, such that

$$
\left\|\partial^{\alpha} \partial_{1} \Theta_{\mathfrak{p}}\right\|_{L^{q}\left(\mathbb{R}^{2}\right)}+\varepsilon_{\mathfrak{p}}\left\|\partial^{\alpha} \partial_{2} \Theta_{\mathfrak{p}}\right\|_{L^{q}\left(\mathbb{R}^{2}\right)} \leq K(q, \alpha),
$$

for any $1<q<+\infty$, any $\alpha \in \mathbb{N}^{2}$ such that $|\alpha| \leq k+1$, and any $\mathfrak{p}$ sufficiently small.

Proof. Inequality (6.18) is a consequence of (4.26). Applying Sobolev embedding theorem to assumption (6.1), we have

$$
\left\|N_{\mathfrak{p}}\right\|_{C^{k}\left(\mathbb{R}^{2}\right)} \leq K(k),
$$

where $K(k)$ is some positive constant, not depending on $\mathfrak{p}$. Therefore, given any $\alpha \in \mathbb{N}^{2}$ such that $|\alpha| \leq k+1$, (4.26) may be written as

$$
\begin{gathered}
\left\|\partial^{\alpha} \partial_{1} \Theta_{\mathfrak{p}}\right\|_{L^{q}\left(\mathbb{R}^{2}\right)}+\varepsilon_{\mathfrak{p}}\left\|\partial^{\alpha} \partial_{2} \Theta_{\mathfrak{p}}\right\|_{L^{q}\left(\mathbb{R}^{2}\right)} \\
\leq K(q, \alpha)\left(\left\|\partial^{\alpha} N_{\mathfrak{p}}\right\|_{L^{q}\left(\mathbb{R}^{2}\right)}+\varepsilon_{\mathfrak{p}}^{2} \sum_{0 \leq \beta<\alpha}\left(\left\|\partial^{\alpha-\beta} \partial_{1} \Theta_{\mathfrak{p}}\right\|_{L^{q}\left(\mathbb{R}^{2}\right)}+\varepsilon_{\mathfrak{p}}\left\|\partial^{\alpha-\beta} \partial_{2} \Theta_{\mathfrak{p}}\right\|_{L^{q}\left(\mathbb{R}^{2}\right)}\right)\right) .
\end{gathered}
$$

Denoting

$$
S_{k}^{q}=\sum_{|\alpha| \leq k+1}\left(\left\|\partial^{\alpha} \partial_{1} \Theta_{\mathfrak{p}}\right\|_{L^{q}\left(\mathbb{R}^{2}\right)}+\varepsilon_{\mathfrak{p}}\left\|\partial^{\alpha} \partial_{2} \Theta_{\mathfrak{p}}\right\|_{L^{q}\left(\mathbb{R}^{2}\right)}\right),
$$

we deduce that

$$
S_{k}^{q} \leq K(q, \alpha)\left(\varepsilon_{\mathfrak{p}}^{2} S_{k}^{q}+\sum_{|\alpha| \leq k+1}\left\|\partial^{\alpha} N_{\mathfrak{p}}\right\|_{L^{q}\left(\mathbb{R}^{2}\right)}\right) .
$$

Combined with assumption (6.1), this provides (6.18) for any $\mathfrak{p}$ sufficiently small.

We now turn to $L^{q}$-estimates of the functions $f_{\mathfrak{p}}$ and $\mathcal{R}_{\varepsilon_{\mathfrak{p}}}^{i, j}$. 
LEMmA 6.6. Let $k \in \mathbb{N}$, and assume that (6.1) holds for any $1<q<+\infty$ and any $\alpha \in \mathbb{N}^{2}$ such that $|\alpha| \leq k$. Then, there exist some positive constants $K(q, \alpha)$, not depending on $\mathfrak{p}$, such that

$\left\|\partial^{\alpha} f_{\mathfrak{p}}\right\|_{L^{q}\left(\mathbb{R}^{2}\right)}+\left\|\partial^{\alpha} \mathcal{R}_{\varepsilon_{\mathfrak{p}}}^{0,2}\right\|_{L^{q}\left(\mathbb{R}^{2}\right)}+\varepsilon_{\mathfrak{p}}\left\|\partial^{\alpha} \mathcal{R}_{\varepsilon_{\mathfrak{p}}}^{1,1}\right\|_{L^{q}\left(\mathbb{R}^{2}\right)}+\varepsilon_{\mathfrak{p}}^{2}\left\|\partial^{\alpha} \mathcal{R}_{\varepsilon_{\mathfrak{p}}}^{2,0}\right\|_{L^{q}\left(\mathbb{R}^{2}\right)} \leq K(q, \alpha)$,

for any $1<q<+\infty$, any $\alpha \in \mathbb{N}^{2}$ such that $|\alpha| \leq k+1$, and any $\mathfrak{p}$ sufficiently small.

ProOF. Lemma 6.6 is a consequence of assumption (6.1), and Lemma 6.5. For instance, applying Leibniz formula to definition (4.34), we have

$$
\left|\partial^{\alpha} f_{\mathfrak{p}}\right| \leq K(\alpha) \sum_{0 \leq \beta \leq \alpha}\left(\left|\partial^{\beta} N_{\mathfrak{p}}\right|\left|\partial^{\alpha-\beta} N_{\mathfrak{p}}\right|+\left|\partial^{\beta} \partial_{1} \Theta_{\mathfrak{p}}\right|\left|\partial^{\alpha-\beta} \partial_{1} \Theta_{\mathfrak{p}}\right|\right),
$$

so that, by (6.1), (6.18), and Hölder inequality,

$$
\left\|\partial^{\alpha} f_{\mathfrak{p}}\right\|_{L^{q}\left(\mathbb{R}^{2}\right)} \leq K(q, \alpha) .
$$

The proof is identical for the function $\mathcal{R}_{\varepsilon_{\mathfrak{p}}}^{1,1}$, which verifies, in view of (4.18) and Leibniz formula,

$$
\left|\partial^{\alpha} \mathcal{R}_{\varepsilon_{\mathfrak{p}}}^{1,1}\right| \leq K(\alpha) \sum_{0 \leq \beta \leq \alpha}\left|\partial^{\beta} N_{\mathfrak{p}}\right|\left|\partial^{\alpha-\beta} \partial_{2} \Theta_{\mathfrak{p}}\right|
$$

Similarly, for $\partial^{\alpha} \mathcal{R}_{\varepsilon_{\mathfrak{p}}}^{2,0}$ and $\partial^{\alpha} \mathcal{R}_{\varepsilon_{\mathfrak{p}}}^{0,2}$, it follows from (6.1), (6.18) and Leibniz formula, that

$$
\begin{gathered}
\left\|\partial^{\alpha} \mathcal{R}_{\varepsilon_{\mathfrak{p}}}^{0,2}\right\|_{L^{q}\left(\mathbb{R}^{2}\right)}+\varepsilon_{\mathfrak{p}}^{2}\left\|\partial^{\alpha} \mathcal{R}_{\varepsilon_{\mathfrak{p}}}^{2,0}\right\|_{L^{q}\left(\mathbb{R}^{2}\right)} \\
\leq K(q, \alpha)\left(1+\varepsilon_{\mathfrak{p}}^{2}\left\|\partial^{\alpha}\left(\frac{\left(\partial_{1} N_{\mathfrak{p}}\right)^{2}}{1-\frac{\varepsilon_{\mathfrak{p}}^{2}}{6} N_{\mathfrak{p}}}\right)\right\|_{L^{q}\left(\mathbb{R}^{2}\right)}+\varepsilon_{\mathfrak{p}}^{4}\left\|\partial^{\alpha}\left(\frac{\left(\partial_{2} N_{\mathfrak{p}}\right)^{2}}{1-\frac{\varepsilon_{\mathfrak{p}}^{2}}{6} N_{\mathfrak{p}}}\right)\right\|_{L^{q}\left(\mathbb{R}^{2}\right)}\right),
\end{gathered}
$$

so that the proof of (6.19) reduces to estimate the $L^{q}$-norms in the left-hand side of (6.20). In view of (6.1), we deduce from Sobolev embedding theorem that

$$
\left\|\partial^{\beta} N_{\mathfrak{p}}\right\|_{L^{\infty}\left(\mathbb{R}^{2}\right)} \leq K(\beta),
$$

for any $\beta \in \mathbb{R}^{2}$ such that $\beta \leq k$ and any $\mathfrak{p}$ sufficiently small. When $|\alpha| \leq k$, the chain rule theorem combined with (6.1) and (6.21) again provides estimates (6.19). When $|\alpha|=k+1$, this argument yields

$$
\varepsilon_{\mathfrak{p}}^{2}\left\|\partial^{\alpha}\left(\frac{\left(\partial_{1} N_{\mathfrak{p}}\right)^{2}}{1-\frac{\varepsilon_{\mathfrak{p}}^{2}}{6} N_{\mathfrak{p}}}\right)\right\|_{L^{q}\left(\mathbb{R}^{2}\right)} \leq K(q, \alpha)\left(1+\varepsilon_{\mathfrak{p}}^{2}\left\|\partial^{\alpha} \partial_{1} N_{\mathfrak{p}}\right\|_{L^{q}\left(\mathbb{R}^{2}\right)}\right) \leq K(q, \alpha),
$$

and

$$
\varepsilon_{\mathfrak{p}}^{4}\left\|\partial^{\alpha}\left(\frac{\left(\partial_{2} N_{\mathfrak{p}}\right)^{2}}{1-\frac{\varepsilon_{\mathfrak{p}}^{2}}{6} N_{\mathfrak{p}}}\right)\right\|_{L^{q}\left(\mathbb{R}^{2}\right)} \leq K(q, \alpha)\left(1+\varepsilon_{\mathfrak{p}}^{4}\left\|\partial^{\alpha} \partial_{2} N_{\mathfrak{p}}\right\|_{L^{q}\left(\mathbb{R}^{2}\right)}\right) \leq K(q, \alpha),
$$

where we have used the estimates in the second line of (6.1) for the second inequalities. Combined with (6.20), this completes the proof of inequality (6.19). 
6.6. Proof of Proposition 6.1. We are now in position to conclude the inductive proof of Proposition 6.1.

Proof of Proposition 6.1. Given any $k \in \mathbb{N}$, we assume that (6.1) holds for any $1<q<+\infty$ and any $\alpha \in \mathbb{N}^{2}$ such that $|\alpha| \leq k$, and consider some index $\gamma \in \mathbb{N}^{2}$ such that $|\gamma|=k+1$. Invoking equation (6.5) and the kernel estimates of Lemma 5.2, we compute

$$
\begin{aligned}
\left\|\partial^{\gamma} \partial_{1} N_{\mathfrak{p}}\right\|_{L^{q}\left(\mathbb{R}^{2}\right)} \leq & K(q)\left(\left\|\partial^{\gamma} f_{\mathfrak{p}}\right\|_{L^{q}\left(\mathbb{R}^{2}\right)}+\varepsilon_{\mathfrak{p}}^{2}\left(\left\|\partial^{\gamma} \mathcal{R}_{\varepsilon_{\mathfrak{p}}}^{2,0}\right\|_{L^{q}\left(\mathbb{R}^{2}\right)}\right.\right. \\
& \left.\left.+\left\|\partial^{\gamma} \mathcal{R}_{\varepsilon_{\mathfrak{p}}}^{1,1}\right\|_{L^{q}\left(\mathbb{R}^{2}\right)}\right)+\varepsilon_{\mathfrak{p}}\left\|\partial^{\gamma} \mathcal{R}_{\varepsilon_{\mathfrak{p}}}^{0,2}\right\|_{L^{q}\left(\mathbb{R}^{2}\right)}\right),
\end{aligned}
$$

and

$$
\begin{gathered}
\left\|\partial^{\gamma} \partial_{2} N_{\mathfrak{p}}\right\|_{L^{q}\left(\mathbb{R}^{2}\right)}+\left\|\partial^{\gamma} \partial_{1}^{2} N_{\mathfrak{p}}\right\|_{L^{q}\left(\mathbb{R}^{2}\right)}+\varepsilon_{\mathfrak{p}}\left\|\partial^{\gamma} \partial_{1} \partial_{2} N_{\mathfrak{p}}\right\|_{L^{q}\left(\mathbb{R}^{2}\right)}+\varepsilon_{\mathfrak{p}}^{2}\left\|\partial^{\gamma} \partial_{2}^{2} N_{\mathfrak{p}}\right\|_{L^{q}\left(\mathbb{R}^{2}\right)} \\
\left.\leq K(q)\left(\left\|\partial^{\gamma} f_{\mathfrak{p}}\right\|_{L^{q}\left(\mathbb{R}^{2}\right)}+\varepsilon_{\mathfrak{p}}^{2}\left\|\partial^{\gamma} \mathcal{R}_{\varepsilon_{\mathfrak{p}}, 0}^{2,0}\right\|_{L^{q}\left(\mathbb{R}^{2}\right)}+\varepsilon_{\mathfrak{p}}\left\|\partial^{\gamma} \mathcal{R}_{\varepsilon_{\mathfrak{p}}}^{1,1}\right\|_{L^{q}\left(\mathbb{R}^{2}\right)}\right)+\left\|\partial^{\gamma} \mathcal{R}_{\varepsilon_{\mathfrak{p}}}^{0,2}\right\|_{L^{q}\left(\mathbb{R}^{2}\right)}\right) .
\end{gathered}
$$

In view of inequalities (6.6), (6.22) and (6.23), and estimates (6.19), assumption (6.1) also holds for $\alpha=\gamma$. This completes the inductive proof of Proposition 6.1 .

\section{Convergence towards (KP I)}

This section is devoted to the proofs of Theorem 2 and Proposition 2. As mentioned above in the introduction, our strategy is to prove that the sequence $\left(\partial_{1} \Theta_{\mathfrak{p}}\right)_{\mathfrak{p}>0}$ is, for $\mathfrak{p}$ sufficiently small, a minimizing sequence for minimization problem $\left(\mathcal{P}_{K P}(\mu)\right)$ We then invoke Proposition 2.1 to obtain the strong convergence of some subsequence towards a function $N_{0}$, which is a solution to minimization problem $\left(\mathcal{P}_{K P}(\mu)\right)$, i.e. a ground state for (KP I). Finally, we improve the convergence using the previous Sobolev estimates.

7.1. Weak convergence towards (KP I). We first use the Sobolev bounds provided by Proposition 3 to establish the weak convergence of some subsequence $\left(N_{\mathfrak{p}_{n}}\right)_{n \in \mathbb{N}}$ to some non-constant solution $N_{0}$ to (SW), as $\mathfrak{p}_{n} \rightarrow 0$.

Proposition 7.1. There exists a subsequence $\left(\mathfrak{p}_{n}\right)_{n \in \mathbb{N}}$, tending to 0 as $n \rightarrow$ $+\infty$, and a non-constant solution $N_{0}$ to (SW) such that, given any $1<q<+\infty$,

$$
N_{\mathfrak{p}_{n}} \rightarrow N_{0} \text { in } W^{1, q}\left(\mathbb{R}^{2}\right) \text {, as } n \rightarrow+\infty .
$$

In particular, given any $0 \leq \gamma<1$, we have

$$
N_{\mathfrak{p}_{n}} \rightarrow N_{0} \text { in } \mathcal{C}^{0, \gamma}(K) \text {, as } n \rightarrow+\infty,
$$

for any compact subset $K$ of $\mathbb{R}^{2}$.

Proof. In view of bounds (11), there exists a subsequence $\left(\mathfrak{p}_{n}\right)_{n \in \mathbb{N}}$, tending to 0 as $n \rightarrow+\infty$, and a function $N_{0}$ such that (7.1) holds for any $1<q<$ $+\infty$. Convergences (7.2) follow by standard compactness theorems. The proof of Proposition 7.1 therefore reduces to prove Lemma 2, i.e. to establish that $N_{0}$ is a non-constant solution to (SW). 
Proof of Lemma 2. Denoting

$$
N_{\mathfrak{p}}^{0}=\frac{1}{2} K_{\varepsilon_{\mathfrak{p}}}^{2,0} \star f_{\mathfrak{p}},
$$

we deduce from (4.33) and Lemma 5.1 that

$$
\begin{aligned}
\left\|N_{\mathfrak{p}}-N_{\mathfrak{p}}^{0}\right\|_{L^{2}\left(\mathbb{R}^{2}\right)} & \leq \varepsilon_{\mathfrak{p}}^{2} \sum_{i+j=2}\left\|K_{\varepsilon_{\mathfrak{p}}}^{i, j} \star \mathcal{R}_{\varepsilon_{\mathfrak{p}}}^{i, j}\right\|_{L^{2}\left(\mathbb{R}^{2}\right)} \\
& \leq \varepsilon_{\mathfrak{p}}^{2}\left\|\mathcal{R}_{\varepsilon_{\mathfrak{p}}}^{2,0}\right\|_{L^{1}\left(\mathbb{R}^{2}\right)}+\varepsilon_{\mathfrak{p}}^{\frac{3}{2}}\left\|\mathcal{R}_{\varepsilon_{\mathfrak{p}}}^{1,1}\right\|_{L^{1}\left(\mathbb{R}^{2}\right)}+\varepsilon_{\mathfrak{p}}^{\frac{1}{2}}\left\|\mathcal{R}_{\varepsilon_{\mathfrak{p}}}^{0,2}\right\|_{L^{1}\left(\mathbb{R}^{2}\right)} .
\end{aligned}
$$

In view of estimates (6.7), (6.8), (6.9) and (6.10), and $L^{q}$-bounds (11), we obtain

$$
\left\|N_{\mathfrak{p}}-N_{\mathfrak{p}}^{0}\right\|_{L^{2}\left(\mathbb{R}^{2}\right)} \leq K \varepsilon_{\mathfrak{p}}^{\frac{1}{2}}
$$

so that

$$
N_{\mathfrak{p}}-N_{\mathfrak{p}}^{0} \rightarrow 0 \text { in } L^{2}\left(\mathbb{R}^{2}\right), \text { as } \mathfrak{p} \rightarrow 0 .
$$

We now claim that, up to some subsequence $\left(\mathfrak{p}_{n}\right)_{n \in \mathbb{N}}$ satisfying (7.2),

$$
N_{\mathfrak{p}_{n}}^{0} \rightarrow \frac{1}{2} K_{0} \star N_{0}^{2} \text { in } L^{2}\left(\mathbb{R}^{2}\right), \text { as } n \rightarrow+\infty .
$$

Invoking the weak $L^{2}$-convergence provided by (7.1), we deduce from (7.3) and (7.4) that the function $N_{0}$ satisfies

$$
N_{0}=\frac{1}{2} K_{0} \star N_{0}^{2}
$$

so that, in view of $(2.3)$, the function $N_{0}$ is solution to (SW).

Finally, in view of (8) and convergences (7.2), we have

$$
N_{0}(0) \geq \frac{3}{5}
$$

so that $N_{0}$ cannot be a constant solution to (SW). This ends the proof of Lemma 2.

We now show Claim (7.4).

Proof of Claim (7.4). Claim (7.4) follows from (7.2) after the following simplification.

STEP 1. We have

$$
N_{\mathfrak{p}}^{0}-\frac{1}{2} K_{0} \star N_{\mathfrak{p}}^{2} \rightarrow 0 \text { in } L^{2}\left(\mathbb{R}^{2}\right), \text { as } \mathfrak{p} \rightarrow 0 .
$$

In view of (4.34), we have

$$
N_{\mathfrak{p}}^{0}-\frac{1}{2} K_{0} \star N_{\mathfrak{p}}^{2}=\left(K_{\varepsilon_{\mathfrak{p}}}^{2,0}-K_{0}\right) \star\left(\frac{1}{3} N_{\mathfrak{p}}^{2}+\frac{1}{6}\left(\partial_{1} \Theta_{\mathfrak{p}}\right)^{2}\right)+\frac{1}{6} K_{0} \star\left(\left(\partial_{1} \Theta_{\mathfrak{p}}\right)^{2}-N_{\mathfrak{p}}^{2}\right)
$$

so that, by Young inequality, and estimates (11),

$$
\left\|N_{\mathfrak{p}}^{0}-\frac{1}{2} K_{0} \star N_{\mathfrak{p}}^{2}\right\|_{L^{2}\left(\mathbb{R}^{2}\right)} \leq K\left(\left\|K_{\varepsilon_{\mathfrak{p}}}^{2,0}-K_{0}\right\|_{L^{2}\left(\mathbb{R}^{2}\right)}+\left\|K_{0}\right\|_{L^{2}\left(\mathbb{R}^{2}\right)}\left\|\partial_{1} \Theta_{\mathfrak{p}}-N_{\mathfrak{p}}\right\|_{L^{2}\left(\mathbb{R}^{2}\right)}\right) .
$$

In view of definitions (2.4) and (4.32), we have

$$
\widehat{K_{\varepsilon_{\mathfrak{p}}}^{2,0}}(\xi) \rightarrow \widehat{K_{0}}(\xi), \text { as } \mathfrak{p} \rightarrow 0,
$$


and

$$
0 \leq \widehat{K_{\varepsilon_{\mathfrak{p}}}^{2,0}}(\xi) \leq \widehat{K_{0}}(\xi)
$$

for any $\varepsilon_{\mathfrak{p}} \geq 0$ and any $\xi \neq 0$. Since $K_{0}$ belongs to $L^{2}\left(\mathbb{R}^{2}\right)$ by Lemma 5.1 , it follows from the dominated convergence theorem that

$$
\int_{\mathbb{R}^{2}}\left|\widehat{K_{\varepsilon_{\mathfrak{p}}}^{2,0}}(\xi)-\widehat{K_{0}}(\xi)\right|^{2} d \xi \rightarrow 0, \text { as } \varepsilon_{\mathfrak{p}} \rightarrow 0 .
$$

Hence, by Plancherel formula, the first term in the right-hand side of (7.5) tends to 0 , as $\mathfrak{p} \rightarrow 0$, whereas the second term also tends to 0 by (4.12). This completes the proof of Step 1.

Invoking Step 1, the proof of Claim (7.4) reduces to

SteP 2. Given some subsequence $\left(\mathfrak{p}_{n}\right)_{n \in \mathbb{N}}$ such that (7.2) holds, we have

$$
K_{0} \star N_{\mathfrak{p}_{n}}^{2} \rightarrow K_{0} \star N_{0}^{2} \text { in } L^{2}\left(\mathbb{R}^{2}\right) \text {, as } n \rightarrow+\infty .
$$

First notice that, in view of (11), there exists some constant $K$, not depending on $n$, such that

$$
\left\|K_{0} \star\left(N_{\mathfrak{p}_{n}}^{2}-N_{0}^{2}\right)\right\|_{L^{2}\left(\mathbb{R}^{2}\right)} \leq\left\|K_{0}\right\|_{L^{2}\left(\mathbb{R}^{2}\right)}\left\|N_{\mathfrak{p}_{n}}^{2}-N_{0}^{2}\right\|_{L^{1}\left(\mathbb{R}^{2}\right)} \leq K,
$$

so that by density of $\mathcal{C}_{c}^{\infty}\left(\mathbb{R}^{2}\right)$ into $L^{2}\left(\mathbb{R}^{2}\right)$, the proof of Step 2 reduces to prove that

$$
\int_{\mathbb{R}^{2}}\left(K_{0} \star\left(N_{\mathfrak{p}_{n}}^{2}-N_{0}^{2}\right)\right) \psi \rightarrow 0, \text { as } n \rightarrow+\infty,
$$

for any function $\psi \in \mathcal{C}_{c}^{\infty}\left(\mathbb{R}^{2}\right)$. Moreover, given any $\delta>0$, the density of $\mathcal{C}_{c}^{\infty}\left(\mathbb{R}^{2}\right)$ into $L^{2}\left(\mathbb{R}^{2}\right)$ also implies the existence of a function $\kappa_{\delta} \in \mathcal{C}_{c}^{\infty}\left(\mathbb{R}^{2}\right)$ such that

$$
\left\|K_{0}-\kappa_{\delta}\right\|_{L^{2}\left(\mathbb{R}^{2}\right)} \leq \delta
$$

Given any function $\psi \in \mathcal{C}_{c}^{\infty}\left(\mathbb{R}^{2}\right)$, this gives by Young inequality,

$\left|\int_{\mathbb{R}^{2}}\left(K_{0} \star\left(N_{\mathfrak{p}_{n}}^{2}-N_{0}^{2}\right)\right) \psi\right| \leq\left|\int_{\mathbb{R}^{2}}\left(\kappa_{\delta} \star\left(N_{\mathfrak{p}_{n}}^{2}-N_{0}^{2}\right)\right) \psi\right|+\delta\left\|N_{\mathfrak{p}_{n}}^{2}-N_{0}^{2}\right\|_{L^{1}\left(\mathbb{R}^{2}\right)}\|\psi\|_{L^{2}\left(\mathbb{R}^{2}\right)}$, which may be written as

$$
\left|\int_{\mathbb{R}^{2}}\left(K_{0} \star\left(N_{\mathfrak{p}_{n}}^{2}-N_{0}^{2}\right)\right) \psi\right| \leq\left|\int_{\mathbb{R}^{2}}\left(\check{\kappa}_{\delta} \star \psi\right)\left(N_{\mathfrak{p}_{n}}^{2}-N_{0}^{2}\right)\right|+K \delta,
$$

denoting $\check{\kappa}_{\delta}(x)=\kappa_{\delta}(-x)$, and invoking (11) and Fubini theorem. Since the function $\check{\kappa}_{\delta} \star \psi$ belongs to $\mathcal{C}_{c}^{\infty}\left(\mathbb{R}^{2}\right)$, we deduce from (7.2) that

$$
\int_{\mathbb{R}^{2}}\left(\check{\kappa}_{\delta} \star \psi\right)\left(N_{\mathfrak{p}_{n}}^{2}-N_{0}^{2}\right) \rightarrow 0, \text { as } n \rightarrow+\infty,
$$

so that (7.6) holds. This completes the proof of Step 2 and of Claim (7.4).

7.2. Convergence of the energies. In order to apply Proposition 2.1 to the family $\left(\partial_{1} \Theta_{\mathfrak{p}}\right)_{\mathfrak{p}>0}$ to deduce its strong convergence in the space $Y\left(\mathbb{R}^{2}\right)$, we first prove

Proposition 7.2. Let $\left(\mathfrak{p}_{n}\right)_{n>0}$ denote some subsequence, tending to 0 as $n$ tends to $+\infty$, such that (7.1) and (7.2) hold. Then, up to some further subsequence, there exists a positive number $\mu_{0}$ such that

$$
E_{K P}\left(\partial_{1} \Theta_{\mathfrak{p}_{n}}\right) \rightarrow \mathcal{E}_{\min }^{K P}\left(\mu_{0}\right), \text { and } \int_{\mathbb{R}^{2}}\left|\partial_{1} \Theta_{\mathfrak{p}_{n}}\right|^{2} \rightarrow \mu_{0}, \text { as } n \rightarrow+\infty .
$$


Proposition 7.2 is a consequence of Lemmas 3 and 4 , so that we first address the proof of Lemma 3.

Proof OF Lemma 3. In view of formulae (4.2) and (4.3), the discrepancy quantity $\Sigma\left(u_{\mathfrak{p}}\right)=\sqrt{2} p\left(u_{\mathfrak{p}}\right)-E\left(u_{\mathfrak{p}}\right)$ may be recast in the slow space variables as

$$
\begin{aligned}
\Sigma\left(u_{\mathfrak{p}}\right) & =-\sqrt{2} \frac{\varepsilon_{\mathfrak{p}}}{144}\left(\int_{\mathbb{R}^{2}}\left(N_{\mathfrak{p}}-\partial_{1} \Theta_{\mathfrak{p}}\right)^{2}+\varepsilon_{\mathfrak{p}}^{2} \int_{\mathbb{R}^{2}}\left(\frac{1}{2}\left(\partial_{1} N_{\mathfrak{p}}\right)^{2}+\frac{1}{2}\left(\partial_{2} \Theta_{\mathfrak{p}}\right)^{2}\right.\right. \\
& \left.\left.-\frac{1}{6} N_{\mathfrak{p}}\left(\partial_{1} \Theta_{\mathfrak{p}}\right)^{2}\right)+\varepsilon_{\mathfrak{p}}^{4} \int_{\mathbb{R}^{2}}\left(\frac{\left(\partial_{2} N_{\mathfrak{p}}\right)^{2}}{4-\frac{4 \varepsilon_{\mathfrak{p}}^{2}}{6} N_{\mathfrak{p}}}+\frac{N_{\mathfrak{p}}\left(\partial_{1} N_{\mathfrak{p}}\right)^{2}}{12-2 \varepsilon_{\mathfrak{p}}^{2} N_{\mathfrak{p}}}-\frac{1}{12} N_{\mathfrak{p}}\left(\partial_{2} \Theta_{\mathfrak{p}}\right)^{2}\right)\right) .
\end{aligned}
$$

Hence, we deduce from Proposition 3 and estimate (4.8) for the function $\partial_{2} \Theta_{\mathfrak{p}}$ that

$$
\begin{aligned}
\Sigma\left(u_{\mathfrak{p}}\right)= & -\sqrt{2} \frac{\varepsilon_{\mathfrak{p}}}{144}\left(\int_{\mathbb{R}^{2}}\left(N_{\mathfrak{p}}-\partial_{1} \Theta_{\mathfrak{p}}\right)^{2}+\varepsilon_{\mathfrak{p}}^{2} \int_{\mathbb{R}^{2}}\left(\frac{1}{2}\left(\partial_{1} N_{\mathfrak{p}}\right)^{2}\right.\right. \\
+ & \left.\left.\frac{1}{2}\left(\partial_{2} \Theta_{\mathfrak{p}}\right)^{2}-\frac{1}{6} N_{\mathfrak{p}}\left(\partial_{1} \Theta_{\mathfrak{p}}\right)^{2}\right)+\underset{\mathfrak{p} \rightarrow 0}{o}\left(\varepsilon_{\mathfrak{p}}^{2}\right)\right) .
\end{aligned}
$$

Let us now recall that the value of $E_{K P}\left(\partial_{1} \Theta_{\mathfrak{p}}\right)$ is given by

$$
E_{K P}\left(\partial_{1} \Theta_{\mathfrak{p}}\right)=\int_{\mathbb{R}^{2}}\left(\frac{1}{2}\left(\partial_{1}^{2} \Theta_{\mathfrak{p}}\right)^{2}+\frac{1}{2}\left(\partial_{2} \Theta_{\mathfrak{p}}\right)^{2}-\frac{1}{6}\left(\partial_{1} \Theta_{\mathfrak{p}}\right)^{3}\right) .
$$

In particular, provided we may prove that

$$
\partial_{1} N_{\mathfrak{p}} \rightarrow \partial_{1}^{2} \Theta_{\mathfrak{p}}, \text { as } \mathfrak{p} \rightarrow 0,
$$

we have, in view of (11) and (4.12),

$$
\int_{\mathbb{R}^{2}}\left(\frac{1}{2}\left(\partial_{1} N_{\mathfrak{p}}\right)^{2}+\frac{1}{2}\left(\partial_{2} \Theta_{\mathfrak{p}}\right)^{2}-\frac{1}{6} N_{\mathfrak{p}}\left(\partial_{1} \Theta_{\mathfrak{p}}\right)^{2}\right)-E_{K P}\left(\partial_{1} \Theta_{\mathfrak{p}}\right) \rightarrow 0, \text { as } \mathfrak{p} \rightarrow 0
$$

Hence, by (7.8),

$$
\Sigma\left(u_{\mathfrak{p}}\right)=-\sqrt{2} \frac{\varepsilon_{\mathfrak{p}}}{144}\left(\int_{\mathbb{R}^{2}}\left(N_{\mathfrak{p}}-\partial_{1} \Theta_{\mathfrak{p}}\right)^{2}+\varepsilon_{\mathfrak{p}}^{2} E_{K P}\left(\partial_{1} \Theta_{\mathfrak{p}}\right)+\underset{\mathfrak{p} \rightarrow 0}{o}\left(\varepsilon_{\mathfrak{p}}^{2}\right)\right) .
$$

We then claim that

$$
\frac{1}{\varepsilon_{\mathfrak{p}}^{2}} \int_{\mathbb{R}^{2}}\left(N_{\mathfrak{p}}-\partial_{1} \Theta_{\mathfrak{p}}\right)^{2} \rightarrow 0, \text { as } \mathfrak{p} \rightarrow 0
$$

which gives (16) using (7.11).

In order to complete the proof of Lemma 3, it only remains to prove Claims (7.9) and (7.12). For Claim (7.9), we invoke equation (4.14) and the Sobolev estimates of Proposition 3. Taking the $L^{2}$-norm of (4.14), we deduce from (11) that

$$
\left\|\partial_{1} N_{\mathfrak{p}}-\partial_{1}^{2} \Theta_{\mathfrak{p}}\right\|_{L^{2}\left(\mathbb{R}^{2}\right)} \leq K \varepsilon_{\mathfrak{p}}
$$

where $K$ is some universal constant. Claim (7.9) follows taking the limit $\mathfrak{p} \rightarrow 0$. Similarly, for Claim 7.12, we take the $L^{2}$-norm of equation (4.13), and obtain by (11),

so that

$$
\left\|N_{\mathfrak{p}}-\partial_{1} \Theta_{\mathfrak{p}}\right\|_{L^{2}\left(\mathbb{R}^{2}\right)} \leq K \varepsilon_{\mathfrak{p}}^{2}
$$

$$
\frac{1}{\varepsilon_{\mathfrak{p}}^{2}} \int_{\mathbb{R}^{2}}\left(N_{\mathfrak{p}}-\partial_{1} \Theta_{\mathfrak{p}}\right)^{2} \leq K \varepsilon_{\mathfrak{p}}^{2} \rightarrow 0, \text { as } \mathfrak{p} \rightarrow 0 .
$$

This concludes the proof of Lemma 3. 
REMARK 7.1. Equivalence (15) is a consequence of inequality (7.10), since it will be proved in the sequel that the quantity $E_{K P}\left(\partial_{1} \Theta_{\mathfrak{p}}\right)$ has a nonzero limit as $\mathfrak{p} \rightarrow 0$.

We now turn to the proof of Lemma 4.

Proof of Lemma 4. Lemma 4 is a consequence of estimate (3) of Theorem 1. Combining (3) with (10) and (16), we obtain

$$
E_{K P}\left(\partial_{1} \Theta_{\mathfrak{p}}\right) \leq-\frac{6912 \mathfrak{p}^{3}}{\mathcal{S}_{K P}^{2} \varepsilon_{\mathfrak{p}}^{3}}+\underset{\mathfrak{p} \rightarrow 0}{o}(1),
$$

so that by formula (4.2),

$$
E_{K P}\left(\partial_{1} \Theta_{\mathfrak{p}}\right) \leq-\frac{1}{54 \mathcal{S}_{K P}^{2}}\left(\int_{\mathbb{R}^{2}} N_{\mathfrak{p}} \partial_{1} \Theta_{\mathfrak{p}}\right)^{3}+\underset{\mathfrak{p} \rightarrow 0}{o}(1) .
$$

In view of (4.12), we have

$$
E_{K P}\left(\partial_{1} \Theta_{\mathfrak{p}}\right) \leq-\frac{1}{54 \mathcal{S}_{K P}^{2}}\left(\int_{\mathbb{R}^{2}}\left(\partial_{1} \Theta_{\mathfrak{p}}\right)^{2}\right)^{3}+\underset{\mathfrak{p} \rightarrow 0}{o}(1) .
$$

On the other hand, it follows from Lemma 2.1 that

$$
E_{K P}\left(\partial_{1} \Theta_{\mathfrak{p}}\right) \geq \mathcal{E}_{\min }^{K P}\left(\int_{\mathbb{R}^{2}}\left(\partial_{1} \Theta_{\mathfrak{p}}\right)^{2}\right)=-\frac{1}{54 \mathcal{S}_{K P}^{2}}\left(\int_{\mathbb{R}^{2}}\left(\partial_{1} \Theta_{\mathfrak{p}}\right)^{2}\right)^{3}
$$

which completes the proof of Lemma 4.

We finally deduce Proposition 7.2 from Lemma 4.

Proof of Proposition 7.2. In view of (4.12) and (7.1), we have

$$
\liminf _{n \rightarrow+\infty} \int_{\mathbb{R}^{2}}\left(\partial_{1} \Theta_{\mathfrak{p}_{n}}\right)^{2} \geq \int_{\mathbb{R}^{2}} N_{0}^{2}
$$

so that we may assume up to some further subsequence, that

$$
\int_{\mathbb{R}^{2}}\left(\partial_{1} \Theta_{\mathfrak{p}_{n}}\right)^{2} \rightarrow \mu_{0}, \text { as } n \rightarrow+\infty
$$

where

$$
\mu_{0} \geq \int_{\mathbb{R}^{2}} N_{0}^{2}>0
$$

Assertion (7.7) is then a consequence of (17), (7.13), and formula (2.7) of $\mathcal{E}_{\min }^{K P}$.

7.3. Strong convergence towards (KP I). We now show Proposition 4. i.e. the strong convergence of the family $\left(N_{\mathfrak{p}}\right)_{\mathfrak{p}>0}$ in $L^{2}\left(\mathbb{R}^{2}\right)$ (up to some subsequence).

Proof of Proposition 4. In view of Proposition 7.2, we may construct a subsequence $\left(\mathfrak{p}_{n}\right)_{n \in \mathbb{N}}$, tending to 0 as $n \rightarrow+\infty$, and some positive number $\mu_{0}$ such that

$$
E_{K P}\left(\partial_{1} \Theta_{\mathfrak{p}_{n}}\right) \rightarrow \mathcal{E}_{\min }^{K P}\left(\mu_{0}\right), \text { and } \int_{\mathbb{R}^{2}}\left|\partial_{1} \Theta_{\mathfrak{p}_{n}}\right|^{2} \rightarrow \mu_{0}, \text { as } n \rightarrow+\infty \text {. }
$$

By Proposition 2.1, up to some further subsequence, there exists some points $\left(a_{n}\right)_{n \in \mathbb{N}}$ and a ground state solution $N_{0}$ to $(2.5)$, with $\sigma=\frac{\mu_{0}^{2}}{\left(\mu^{*}\right)^{2}}$, such that

$$
\partial_{1} \Theta_{\mathfrak{p}_{n}}\left(\cdot-a_{n}\right) \rightarrow N_{0} \text { in } Y\left(\mathbb{R}^{2}\right), \text { as } n \rightarrow+\infty .
$$


By (4.12), we are led to

$$
N_{\mathfrak{p}_{n}}\left(\cdot-a_{n}\right) \rightarrow N_{0} \text { in } L^{2}\left(\mathbb{R}^{2}\right), \text { as } n \rightarrow+\infty .
$$

Invoking Proposition 7.1 for the subsequence $\left(N_{\mathfrak{p}_{n}}\left(\cdot-a_{n}\right)\right)_{n \in \mathbb{N}}$, there exists a nonconstant solution $\tilde{N}_{0}$ to (SW) such that weak convergences (7.1) hold, up to some further subsequence. In particular, by $(7.14), N_{0}=\tilde{N}_{0}$, so that $N_{0}$ is a ground state of speed 1 of (KP I).

In order to complete the proof of Proposition 4, it is now necessary to drop the invariance by translation, i.e. to prove that convergences in $Y\left(\mathbb{R}^{2}\right)$ and in $L^{2}\left(\mathbb{R}^{2}\right)$, also hold for the sequences $\left(\partial_{1} \Theta_{\mathfrak{p}_{n}}\right)_{n \in \mathbb{N}}$, respectively $\left(N_{\mathfrak{p}_{n}}\right)_{n \in \mathbb{N}}$. Assuming first that, up to some further subsequence, there exists some number $a$ such that

$$
a_{n} \rightarrow a, \text { as } n \rightarrow+\infty
$$

we obtain that

$$
\partial_{1} \Theta_{\mathfrak{p}_{n}} \rightarrow N_{0}(\cdot+a) \text { in } Y\left(\mathbb{R}^{2}\right) \text {, and } N_{\mathfrak{p}_{n}} \rightarrow N_{0}(\cdot+a) \text { in } L^{2}\left(\mathbb{R}^{2}\right) \text {, as } n \rightarrow+\infty,
$$

using the continuity of the map $a \mapsto \psi(\cdot-a)$ from $\mathbb{R}$ to any space $L^{q}\left(\mathbb{R}^{2}\right)$ (with $1<q<+\infty)$. Since the function $x \mapsto N_{0}(x+a)$ is still a ground state of speed 1 of (KP I), this completes the proof of Proposition 4.

Hence, it remains to prove that the sequence $\left(a_{n}\right)_{n \in \mathbb{N}}$ contains some bounded subsequence. Assuming by contradiction that this is false, we may construct some subsequence, still denoted $\left(a_{n}\right)_{n \in \mathbb{N}}$, such that

$$
a_{n} \rightarrow+\infty, \text { as } n \rightarrow+\infty \text {. }
$$

In view of (8) and (11), there exists some positive number $\delta$, not depending on $n$, such that

$$
\int_{B(0,1)} N_{\mathfrak{p}_{n}}^{2} \geq 2 \delta
$$

for any $n$ sufficiently large. By (7.14), we also have

$$
\int_{B(0,1)}\left|N_{0}\left(x+a_{n}\right)-N_{\mathfrak{p}_{n}}(x)\right|^{2} d x \rightarrow 0, \text { as } n \rightarrow+\infty,
$$

so that

$$
\int_{B(0,1)}\left|N_{0}\left(x+a_{n}\right)\right|^{2} d x \geq \delta
$$

for any $n$ sufficiently large. However, it is proved in [18] that there exists some positive constant $K$ such that

$$
N_{0}(x) \leq \frac{K}{1+|x|^{2}}, \forall x \in \mathbb{R}^{2},
$$

so that

$$
\frac{10 K}{1+\left|a_{n}\right|^{2}} \geq \delta
$$

for any $n$ sufficiently large. This provides a contradiction to (7.15) and completes the proof of Proposition 4. 
7.4. Proofs of Theorem 2 and Proposition 2. We finally conclude the proofs of our main theorems.

Proof of Theorem 2. In view of Propositions 3 and 4 , given any $k \in \mathbb{N}$ and any $1<q<+\infty$, the family $\left(N_{\mathfrak{p}}\right)_{\mathfrak{p}>0}$ is bounded, uniformly with respect to $\mathfrak{p}$ small, in $W^{k, q}\left(\mathbb{R}^{2}\right)$, and converges, up to some subsequence, to some ground state $N_{0}$ of (KP I) in the space $L^{2}\left(\mathbb{R}^{2}\right)$, as $\mathfrak{p} \rightarrow 0$. Hence, by standard interpolation theorem, it actually converges to $N_{0}$ in $W^{k, q}\left(\mathbb{R}^{2}\right)$. This concludes the proof of Theorem 2 .

Proof of Proposition 2. The proof is identical to the proof of Theorem 2, considering the function $\partial_{1} \Theta_{\mathfrak{p}}$ instead of $N_{\mathfrak{p}}$, and noticing that $Y\left(\mathbb{R}^{2}\right)$ continuously embeds into $L^{2}\left(\mathbb{R}^{2}\right)$.

ACKNOWLEDGEMENTs. The first and second authors acknowledge support from the ANR project JC05-51279, "Équations de Gross-Pitaevskii, d'Euler, et phénomènes de concentration", of the French Ministry of Research.

\section{References}

[1] M.J. Ablowitz and H. Segur. On the evolution of packets of water waves. J. Fluid. Mech., 92(4):691-715, 1979

[2] F. Béthuel, P. Gravejat, and J.-C. Saut. Existence and properties of travelling waves for the Gross-Pitaevskii equation. In A. Farina and J.-C. Saut, editors, Stationary and time dependent Gross-Pitaevskii equations, Contemp. Math. Amer. Math. Soc., Providence, RI, 2008.

[3] F. Béthuel, P. Gravejat, and J.-C. Saut. Travelling waves for the Gross-Pitaevskii equation II. Commun. Math. Phys., in press, 2008.

[4] F. Béthuel, P. Gravejat, J.-C. Saut, and D. Smets. Orbital stability of the black soliton for the gross-pitaevskii equation. Indiana Univ. Math. J, in press, 2008.

[5] F. Béthuel, G. Orlandi, and D. Smets. Vortex rings for the Gross-Pitaevskii equation. J. Eur. Math. Soc., 6(1):17-94, 2004

[6] F. Béthuel and J.-C. Saut. Travelling waves for the Gross-Pitaevskii equation I. Ann. Inst. Henri Poincar, Physique Thorique, 70(2):147-238, 1999.

[7] D. Chiron. Travelling waves for the Gross-Pitaevskii equation in dimension larger than two. Nonlinear Anal., 58(1-2):175-204, 2004.

[8] C. Coste. Nonlinear Schrödinger equation and superfluid hydrodynamics. Eur. Phys. J. B, $1: 245-253,1998$

[9] A. de Bouard and J.-C. Saut. Remarks on the stability of generalized KP solitary waves. In Mathematical problems in the theory of water waves (Luminy, 1995), volume 200 of Contemp. Math., pages 75-84. Amer. Math. Soc., Providence, RI, 1996.

[10] A. de Bouard and J.-C. Saut. Solitary waves of generalized Kadomtsev-Petviashvili equations. Ann. Inst. Henri Poincar, Analyse Non Linaire, 14(2):211-236, 1997.

[11] A. de Bouard and J.-C. Saut. Symmetries and decay of the generalized KadomtsevPetviashvili solitary waves. SIAM J. Math. Anal., 28(5):1064-1085, 1997.

[12] A. Farina. From Ginzburg-Landau to Gross-Pitaevskii. Monatsh. Math., 139:265-269, 2003.

[13] V.L. Ginzburg and L.P. Pitaevskii. On the theory of superfluidity. Sov. Phys. JETP, 34:1240, 1958.

[14] P. Gravejat. Limit at infinity for travelling waves in the Gross-Pitaevskii equation. $C . R$. Math. Acad. Sci. Paris, 336(2):147-152, 2003.

[15] P. Gravejat. A non-existence result for supersonic travelling waves in the Gross-Pitaevskii equation. Commun. Math. Phys., 243(1):93-103, 2003.

[16] P. Gravejat. Decay for travelling waves in the Gross-Pitaevskii equation. Ann. Inst. Henri Poincar, Analyse Non Linaire, 21(5):591-637, 2004.

[17] P. Gravejat. Limit at infinity and nonexistence results for sonic travelling waves in the GrossPitaevskii equation. Differential Integral Equations, 17(11-12):1213-1232, 2004.

[18] P. Gravejat. Asymptotics of the solitary waves for the generalised Kadomtsev-Petviashvili equations. Disc. Cont. Dynam. Syst., 21(3):835-882, 2008. 
[19] E.P. Gross. Hydrodynamics of a superfluid condensate. J. Math. Phys., 4(2):195-207, 1963.

[20] S.V. Iordanskii and A.V. Smirnov. Three-dimensional solitons in He II. JETP Lett., 27(10):535-538, 1978.

[21] C.A. Jones, S.J. Putterman, and P.H. Roberts. Motions in a Bose condensate V. Stability of solitary wave solutions of nonlinear Schrödinger equations in two and three dimensions. $J$. Phys. A, Math. Gen., 19:2991-3011, 1986.

[22] C.A. Jones and P.H. Roberts. Motions in a Bose condensate IV. Axisymmetric solitary waves. J. Phys. A, Math. Gen., 15:2599-2619, 1982.

[23] Y.S. Kivshar and B. Luther-Davies. Dark optical solitons: physics and applications. Phys. Rep., 298:81-197, 1998.

[24] E.A. Kuznetsov and V.E. Zakharov. Multi-scales expansion in the theory of systems integrable by the inverse scattering transform. Phys. D, 18(1-3):455-463, 1986.

[25] D. Lannes and J.-C. Saut. Weakly transverse Boussinesq equations and the KadomtsevPetviashvili approximation. Nonlinearity, 19:2853-2875, 2007.

[26] P.I. Lizorkin. On multipliers of Fourier integrals in the spaces $L_{p, \theta}$. Proc. Steklov Inst. Math., 89:269-290, 1967.

[27] L.P. Pitaevskii. Vortex lines in an imperfect Bose gas. Sov. Phys. JETP, 13(2):451-454, 1961.

[28] É. Tarquini. A lower bound on the energy of travelling waves of fixed speed for the GrossPitaevskii equation. Monatsh. Math., 151(4):333-339, 2007.

Laboratoire Jacques-Louis Lions, Université Pierre et Marie Curie, Boîte Courrier 187, 75252 Paris Cedex 05, France

E-mail address: bethuel@ann.jussieu.fr

Centre de Recherche en Mathématiques de la Décision, Université Paris Dauphine, Place du Maréchal De Lattre De Tassigny, 75775 Paris Cedex 16, France

E-mail address: gravejat@ceremade.dauphine.fr

Laboratoire de Mathématiques, Université Paris Sud, Bâtiment 425, 91405 Orsay Cedex, France

E-mail address: Jean-Claude.Saut@math.u-psud.fr 